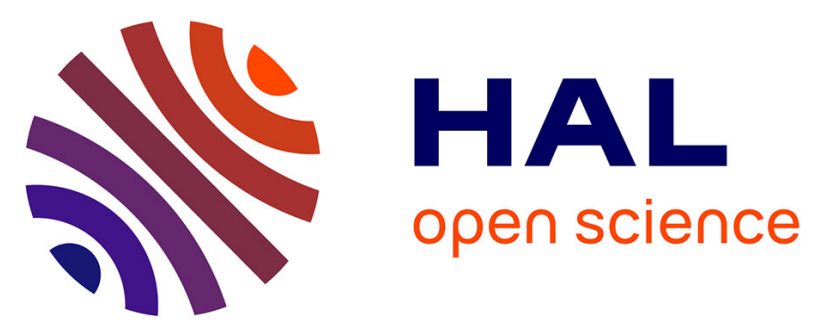

\title{
A robust optimization for a home health care routing and scheduling problem with consideration of uncertain travel and service times
}

Yong Shi, Toufik Boudouh, Olivier Grunder

\section{- To cite this version:}

Yong Shi, Toufik Boudouh, Olivier Grunder. A robust optimization for a home health care routing and scheduling problem with consideration of uncertain travel and service times. Transportation Research Part E: Logistics and Transportation Review, 2019, 128, pp.52 - 95. 10.1016/j.tre.2019.05.015 . hal03480692

\section{HAL Id: hal-03480692 \\ https://hal.science/hal-03480692}

Submitted on 20 Dec 2021

HAL is a multi-disciplinary open access archive for the deposit and dissemination of scientific research documents, whether they are published or not. The documents may come from teaching and research institutions in France or abroad, or from public or private research centers.
L'archive ouverte pluridisciplinaire HAL, est destinée au dépôt et à la diffusion de documents scientifiques de niveau recherche, publiés ou non, émanant des établissements d'enseignement et de recherche français ou étrangers, des laboratoires publics ou privés.

\section{(c) (1) $\$$}

Distributed under a Creative Commons Attribution - NonCommerciall 4.0 International 


\title{
A robust optimization for a home health care routing and scheduling problem with consideration of uncertain travel and service times
}

\author{
Yong Shi ${ }^{\mathrm{a}}$, Toufik Boudouh ${ }^{\mathrm{b}}$, Olivier Grunder ${ }^{\mathrm{a}, *}$
}

${ }^{a}$ Nanomedicine Lab, Univ. Bourgogne Franche-Comté, UTBM, Rue Thierry Mieg, 90010 Belfort, France. ${ }^{b}$ ICB UMR 6303, CNRS, Univ. Bourgogne Franche-Comté, UTBM, Rue Thierry Mieg, 90010 Belfort, France.

\begin{abstract}
In today's competitive environment, one of the most critical objectives for Home Health Care (HHC) companies is to meet the demand of patients in a timely fashion. According to the feedback from HHC companies, caregivers have to deal with some uncertainties when carrying out a given schedule to visit their patients. However, a majority of the previous work only considers the deterministic models which ignore the uncertainties, and solutions obtained by these deterministic models are usually less robust in case of any possible changes in practical situations. Inspired by this point, in this work, we formulate a model for an HHC Routing and Scheduling Problem with taking into account uncertain travel and service times, from the perspective of Robust Optimization (RO) . Specifically, the non-deterministic variables are defined based on the theory of budget uncertainty, and then the arrival time of each caregiver is rewritten as a complicated recursive function. After that, Gurobi Solver, Simulated Annealing, Tabu Search, and Variable Neighborhood Search are adapted to solve the model respectively. Finally, a series of experiments have been performed to validate the proposed models and algorithms. Experimental results from Monte Carlo simulation highlight the strength of considering uncertainties when modeling the problem. Additional, the influences of other characters in instances, like the width of time-window, distributed location have also been empirically analyzed. Finally, the comparison performed between the solutions obtained by the stochastic model and the RO model also demonstrates the advantage of the RO model. This work provides a valuable framework for HHC companies to make a robust schedule when arranging the caregivers.
\end{abstract}

\footnotetext{
${ }^{*}$ Corresponding author. Tel:+33-03-84583329

Email addresses: shiyonghbwh@gmail.com, yong.shi@utbm.fr (Yong Shi), toufik.boudouh@utbm.fr (Toufik
} Boudouh), olivier.grunder@utbm.fr (Olivier Grunder) 


\title{
A robust optimization for a home health care routing and scheduling problem with consideration of uncertain travel and service times
}

\begin{abstract}
In today's competitive environment, one of the most critical objectives for Home Health Care (HHC) companies is to meet the demand of patients in a timely fashion. According to the feedback from HHC companies, caregivers have to deal with some uncertainties when carrying out a given schedule to visit their patients. However, a majority of the previous work only considers the deterministic models which ignore the uncertainties, and solutions obtained by these deterministic models are usually less robust in case of any possible changes in practical situations. Inspired by this point, in this work, we formulate a model for an HHC Routing and Scheduling Problem with taking into account uncertain travel and service times, from the perspective of Robust Optimization (RO) . Specifically, the non-deterministic variables are defined based on the theory of budget uncertainty, and then the arrival time of each caregiver is rewritten as a complicated recursive function. After that, Gurobi Solver, Simulated Annealing, Tabu Search, and Variable Neighborhood Search are adapted to solve the model respectively. Finally, a series of experiments have been performed to validate the proposed models and algorithms. Experimental results from Monte Carlo simulation highlight the strength of considering uncertainties when modeling the problem. Additional, the influences of other characters in instances, like the width of time-window, distributed location have also been empirically analyzed. Finally, the comparison performed between the solutions obtained by the stochastic model and the RO model also demonstrates the advantage of the RO model. This work provides a valuable framework for HHC companies to make a robust schedule when arranging the caregivers.
\end{abstract}

Keywords: Home Health Care, Robust Optimization, Time Window, Vehicle routing problem. 2010 MSC: 90B06, 90C70 


\section{Introduction}

Home health care (HHC) is a wide range of health care services that can be given in one's home for an illness or injury. This service is usually less expensive, more convenient, and just as effective as one can get in a hospital (Alodhayani, 2017). A lot of common health care services such as

5 wound-care, injection, and elder-care could be provided by HHC companies (Tyan, 2010). HHC service can be viewed as an essential form of supplement for health care in developed countries. Taking this service can make the patient stay at home for treatment, and relieve the resource shortage caused by the limited number of hospital beds. In recent years, the health care industry has become one of the largest sectors of the economy in Europe and North America. With the increase of the globally aging problem, there is no doubt that the HHC industry will continuously develop rapidly.

In today's competitive environment, the most critical objective for HHC company is to meet the demand of patients in a timely fashion. According to the feedback from HHC companies, two major operational issues are often encountered. One is delayed service for patients, and another one is an enormous operational cost for HHC companies. Notably, the delay of service may not only bring the tardiness penalty due to the patient dissatisfaction but may also lead to the improper effects on treatment. When dealing with decreasing the cost, we find that one of the most significant challenges in HHC domain is how to utilize the limited resources (both labor resources and equipment) efficiently. Consequently, optimization of HHC Routing and Scheduling Problem (HHCRSP) Fikar \& Hirsch (2017) has become an essential issue for decreasing operational cost, as well as improve delayed service.

Based on the considerable works related to the HHCRSP(Fikar \& Hirsch, 2017), we can summarize the basic procedures of HHC service into three phases. First, an HHC company collects information from patients. Secondly, the decision-makers in the HHC company make an appropriate schedule with entirely taking into account the data collected and the limited resources (labor resources and equipment). Thirdly, the caregivers perform the schedule by driving a car to visit the patients on his/her list, however, they have the authority to adjust little change in case of any changes in practical situations.

Travel and Service Times (TST) are key elements when scheduling the HHC service. According 
have to deal with various types of uncertainty involved in TST. Specifically, the common factors, like varying road conditions, rush hours, weather conditions and driving skills, are always lead to the uncertainty of the travel time. While, the service time for each patient is not always fixed as we estimated due to practical reasons, such as diagnosing time, as well as parking situations. In the third stage, despite the caregivers have the authority to adjust the strategy for visiting patients in case the uncertainty encounters, the strategy may not be optimal to the current situation.

Service quality would be quite poor or even risky, if we neglect the uncertain TST in the planning stage. According to Kuo et al. (2016), the uncertainties may lead to infeasible routes, delayed service or even risky solutions. On the one hand, excessive delay service will reduce customer satisfaction, thus losing the advantage in business competition. On the other hand, some diseases are very time sensitive and must be serviced within a specified time. If delayed, it will have a negative impact on the health of the patient. For example, health care services at patients are highly time-sensitive, e.g., the provision of insulin injections, delays may result in severe consequences for the patients 45 (Fikar \& Hirsch, 2017). Besides, in these situations, TST mostly depend on the practical traffic environment and current weather, whose values are difficult to forecast in advance by the common methods. Therefore, it is of great significance to consider the robustness of the solution when modeling the vehicle routing scheduling in HHC.

Additionally, in the practice of HHC, different patients often come up with different types of demand, such as injections and physical therapy, which require different types of medical skills and qualifications (Akjiratikarl et al., 2007). As a profit-making organization, it is difficult for HHC companies to have enough money to provide the training of all the medical skills for each caregiver. Therefore, to make full use of the limited resources, HHC companies tend to divide caregivers into different levels according to their skills and qualifications. To guarantee the quality of service, caregivers with high-level skills can serve the patients who require low-level demands, while caregivers with low-level skills cannot provide any services to patients with high-level demand. The skill requirement constraints were firstly proposed by Yuan et al. (2015).

In an attempt to find an efficient routing and scheduling strategy for real-world application of HHC, we study the HHCRSP with skill-requirement by taking into account the TST uncertainties. Several techniques can be used for formulating optimization problems when dealing with uncertainties, such as stochastic programming with recourse (Li et al., 2010; Shi et al., 2018; Zhang et al., 2019), robust optimization (Hu et al., 2018), etc. 
The robust optimization method assumes that the uncertain parameters belong to a limited set of uncertainties. For a very general set of uncertainties, the resulting robust counterpart can have a complexity that is comparable to the original problem. For example, a linear program (LP) with uncertain parameters belongs to a set of polyhedral uncertainties with deterministic issues, which are LPs of polynomials of the size of the original problem (Ben-Tal \& Nemirovski, 1999). Our work addresses the HHCRSP from the perspective of robust optimization (RO) model, from which, we attempt to find a robust strategy for an HHC company when arranging the HHC service. To our best knowledge, there is no research investigated HHCRSP with uncertain travel and service times, from the perspective of robust optimization.

The studied problem is quite complicated, due to the reason that it has the attribute of vehicle routing problem, skill-requirement assignment, and robust optimization. To solve the problem, we develop a Simulated Annealing (SA), a Variable Neighborhood Search (VNS) and a Tabu Search 75 (TS) respectively.

This work contributes to the home health care routing problem with the following aspects. (1) A Robust optimization model for home health care routing problem with skill requirement and travel and service uncertainty has been proposed. (2) To solve the problem, we reduced the Robust model to the deterministic model, then Gurobi, SA, VNS, and TS have been adapted to solve the deterministic model directly. (3) TS has been used to solve the robust model. (4) Numerical experimental results have highlighted the advantage of taking into account the uncertainties.

The remainder of the chapter is organized as follows. Section 2 summarizes the recent work related to our problems, and section 3 presents the mathematical model. Proposed approaches are explicitly illustrated in Section 4. After that, a series of experiments are discussed in Section 5. Finally, the paper terminals with conclusions and perspectives.

\section{Literature review}

Di Mascolo et al. (2017) summarized that most of the studies involved in HHC routing and scheduling problems are closely related to the Vehicle Routing Problem (VRP), which is a fundamental issue in transportation planning and logistics. So, in the literature section, we firstly discuss some of the recent work related to VRP, then we analyze deterministic HHCRSP. Finally, uncertain models for HHCRSP are investigated. 
The basic model of VRP is called Capacitated VRP (CVRP), which is an extension of the wellknown traveling salesman problem. CVRP is a classical combinatorial optimization and integer programming problem. The main objectives of the CVRP is to find the optimal routes with multiple

a widespread concern from researchers, because these models have vast applications in many fields, such as retail industry, parcel delivery and home health care services. On the other side, due to the nature of these problems are NP-hard, which is very challenging to be solved, many researchers have developed algorithms for solving them. These algorithms can be divided into heuristic algorithms and exact algorithms.

Wang et al. (2018) addressed the cooperation strategy for the green pickup and delivery problem. In this study, they analyzed the situations in which compensation is needed and develop the lower bound of the compensation. Further, they proposed an exact method to calculate the actual compensation and the profit distribution based on cooperative game theory. The proposed exact method also applied for solving largest scale instance in Li \& Lim benchmarks. Yu et al. (2019) proposed an improved branch \& price algorithm to accurately solve the heterogeneous green fleet vehicles routing problem with time windows. Sun et al. (2019) designed the first exact algorithm for solving a variant of the heterogeneous GVRP. This exact algorithm is based on a set partitioning model and the key characteristics of its optimal solution.

On the other hand, Lai et al. (2016) developed a tabu search heuristic that efficiently handles the parallel arcs for solving a time-constrained heterogeneous vehicle routing problem on a multigraph. Luo et al. (2016) proposed an adaptive large neighborhood search heuristics for the vehicle routing problem with stochastic demands and weight-related cost. Li et al. (2018) addressed the generalized rollon-rolloff vehicle routing problem, which is formulated by a mixed integer linear programming model. The Benders decomposition algorithm involving Pareto-optimal cuts and Benders decomposition-callback implementation, and a two-stage heuristic involving the savings algorithm followed by a local search phase is provided.

$\mathrm{HHC}$ has arisen widespread attention in the last decade, and the majority of the work is performed from the perspective of the medical skills, medical equipment, ethics, and operations management. This section reviews the HHC from the perspective of operational management (OM), specifically, HHCRSP is one of the most essential branches in the study of OM.

Begur et al. (1997) are among the earliest to investigate the issue of HHCRSP, in their work, a 
decision support system for nurse scheduling in HHC has been presented. Mankowska et al. (2014) tackled daily planning of HHC service. The plan takes into account the individual service demand of patients, the personal qualifications of the staff and any interdependencies between the different service operations. Nickel et al. (2012) considered the HHCRSP as two parts. Firstly, they consider the HHCRSP which seeks for a weekly optimal plan. Secondly, in practice, a master schedule is generated from the incorporate operational changes.

In the past five years, HHCRSP has become one of the hottest research points in the area of operations management. Liu et al. (2013) considered a vehicle scheduling problem encountered in home health care logistics. It concerns the delivery of drugs and medical devices from the home care company's pharmacy to patients' homes, delivery of specific drugs from a hospital to patients, pickup of biosamples and unused drugs and medical devices from patients. After that, Liu et al. (2014) further studied a periodic vehicle routing problem encountered in home health care (HHC) logistics. It extends the classical Periodic Vehicle Routing Problem with Time Windows (PVRPTW) to three types of demands of patients at home. A complicated Tabu Search algorithm, integrated different local search schemes were designed to solve the proposed model. To further improve the obtained optimal solution, they utilized the strategy with both feasible and infeasible in the local search.

Decerle et al. (2018) studied the multi-objective HHC problem with the taking in to account applicability of the planning. To solve the proposed issue, a memetic algorithm is developed according to the constraints of the model. Fathollahi-Fard et al. (2018) addressed the bi-objective green $\mathrm{HHC}$ with consideration of green emission and environmental pollution.

Even though considerable works have been addressed in HHCRSPs, only a few works have considered the uncertainties in HHC. The recent works on the HHC with consideration of uncertainties are summarized in Table 1.

Yuan et al. (2015) addressed a HHCSRP with stochastic service times and skill requirements. A stochastic programming model with recourse is proposed to formulate the problem in which the expected penalty for late arrival at patients is considered. Liu et al. (2018) studied the HHCRSP by considering the caregiver's travel times and service times for patients are stochastic. A chance constraint is introduced to ensure the on-time service probability for the patients. Such stochastic traveling and service time and the chance constraint further complicate the problem. In their paper, a route-based mathematical model is introduced. A branch-and-price (B\&P) algorithm 
and a discrete approximation method are combined to solve the problem. Herein, effective label algorithms are designed to generate negative reduced cost routes. The efficiency of the algorithm are improved by employing three acceleration strategies. The experiments on test instances validate the performances of the proposed $\mathrm{B} \& \mathrm{P}$ algorithm and demonstrate the necessity of considering the stochastic of travel times of home-caregiver and service times to the patients.

Lanzarone \& Matta (2014) investigated a robust strategy for home care optimization problem. Specifically, they studied an analytical structural policy to address the issue of nurse-to-patient assignment in home care. This policy explains the randomness of requests from patients already assigned to nurses and requests from new patients requiring assignments. Finally, this policy was compared to other previously developed approaches and analyzed empirically. The proposed model in this study differs with the one proposed by Lanzarone \& Matta (2014) in the following aspects. (1) Our model considers a more general issue in routing and scheduling problem of HHC logistics, which covers travel and service times, skill-requirement constraints. However, Lanzarone \& Matta (2014) just considered a nurse-to-patient assignment problem, without considering patients' time window and skill-requirement assignment, which are essential factors in HHC services. (2) Lanzarone \& Matta (2014) only gave the policy to enhance the robustness of the operational performance of home care, instead of formulating a robust optimization model. But, we formulate our problem by robust optimization technique.

Agra et al. (2013) are among the earliest researchers to address a robust optimization problem for VRP with time windows. They proposed two formulations for the robust optimization problem, which were based on different robust approaches. However, the study of Agra et al. (2013) only considers the uncertainty of travel time. Hu et al. (2018) addressed a robust optimization model of vehicle routing problem with taking into the uncertain demand and travel times. In the VRPs, demand belongs to the capacity constraints, while travel time involves time-window constraints. The two constraints are relatively independently to a certain degree. In this study, the robust home health care problem by considering the uncertain travel and service times simultaneously was investigated. In the proposed model, travel and service times are two independent variables, which show up in the same constraints. So, the problem considered in this study, superposition of the uncertain travel and service times makes the robust version of time-window more complicated than the model proposed by $\mathrm{Hu}$ et al. (2018). Besides, our model also considered the characteristic of HHC logistics by describing the skill-requirements constraints. 
Table 1: Recent research on HHCRSP with considering uncertainties

\begin{tabular}{llll}
\hline Papers & factors & Modeling approach & Solving Method \\
\hline Rodriguez et al. (2015) & uncertain demand & stochastic programming & Branch \& Cut \\
Shi et al. (2017) & fuzzy demand & fuzzy chance constraint programming & Hybrid genetic algorithm \\
Yuan et al. (2015) & stochastic service time & stochastic programming & Branch \& Price \\
Shi et al. (2018) & stochastic travel and service times & stochastic programming model with recourse & Simulated annealing \\
\hline
\end{tabular}

From Table 1, we can find that researchers often use two-stage stochastic programming or a chance-constrained programming model to solve these problems. However, both methods have two distinct drawbacks. (1) These methods assume that the distribution of known parameters, however, in real-life, the history data is inferior to estimate the parameter. (2) Neither of these modeling methods considers the robustness of the solution as a goal. So, these do not guarantee the anti-interference of the final solution.

To sum up, our review of the abundant literature reveals the recent research related to HHCRSP. A majority of work involved HHCRSP only considers the deterministic model without considering the commonly encountered uncertainties. Consequently, the decision made from these solutions show less robust in practical situations. Even though a few works have been done on uncertainties in HHCRSP, they did not consider the problem from the perspective of Robust Optimization. Thus, in this paper, we investigate an HHCRSP with taking into account the uncertain travel and service times simultaneously from the perspective of robustness optimization.

\section{Mathematical model}

Section 3.1 briefly illustrates the assumptions for the studied problem. In section 3.2 , the deterministic MIP model is proposed to describe the HHCRSP with skill requirements. After that, in section 3.3, we develop the constraints of travel and service time uncertainties by utilizing the theory of budget. Finally, the robust optimization model for HHCRSP with skill requirements and travel and service time uncertainties is proposed in section 3.4.

\subsection{Assumptions}

${ }_{205}$ (1) Each caregiver starts a journey from the depot and ends up the trip at the laboratory.

(2) The caregivers have several different levels in term of their skills and qualifications.

(3) The travel time between every two vertexes is uncertain . 
(4) Each caregiver has a maximum limitation number of the patients in his/her task list.

(5) The service time for each patient is uncertain.

\subsection{MIP Model}

Some frequently used notations are introduced before the model construction.

$V$ : set of all vehicles.

$K$ : the number of available vehicles in set $V$.

$C$ : set of all patients.

$i=0,1,2, \ldots, n, n+1$ : the index of patients. Especially, $i=0$ represents the index of unique depot, and $n+1$ depicts the lab.

$N$ : set of all patients, namely $N=C \cup\{0\} \cup\{n+1\}$.

$\left[a_{i}, b_{i}\right]$ : the time window for patient $i$. Especially, when $i=0$ and $i=n+1, a_{i}$ is the opening time of the depot, while $b_{i}$ is the closing time of the laboratory.

$c f_{k}$ : the fixed cost for $k$ th caregiver.

$\sigma$ : the weight to balance the fixed cost of caregivers and the transportation cost.

$d_{i}$ : the level of service for the $i$ th patient's demand.

$D_{k}$ : the level of skills for the $k$ th caregiver.

$Q$ : the maximum number of patients could be visited by each caregiver.

$c_{i j}$ : the transportation cost between patient $i$ and patient $j$.

$t_{i j}$ : the travel time between patients $i$ and $j$ for a single trip.

$t_{i}$ : the service time for patient $i$. Here we need to pay more attention to distinguish between $t_{i}$ and $t_{i j}$.

decision variables:

$x_{i j k}= \begin{cases}1, & \text { if vehicle } k \text { travels from node } i \text { to node } j, \text { in which } i \neq j ; \\ 0, & \text { otherwise. }\end{cases}$

$s_{i k}$ : the beginning service time of patient $i$.

The MIP model can be formulated as:

$$
\text { Deterministic Model: } \min \sigma \cdot \sum_{k \in V} c f_{k} \sum_{j \in C} x_{0 j k}+\sum_{k \in V} \sum_{i \in N} \sum_{j \in N} c_{i j} x_{i j k}
$$


s.t.

$$
\begin{gathered}
\sum_{k \in V} \sum_{j \in N} x_{i j k}=1, \forall i \in C, \\
\sum_{i \in N} x_{i h k}-\sum_{j \in N} x_{h j k}=0, \forall h \in C ; k \in V, \\
\sum_{j \in C} x_{0 j k} \leq 1, \forall k \in V, \\
\sum_{j \in C} x_{j(n+1) k} \leq 1, \forall k \in V, \\
\sum_{i \in N} \sum_{j \in N} x_{i j k} \leq Q, \forall k \in V, \\
d_{i} \sum_{j \in N} x_{i j k} \leq D_{k}, \forall k \in V, i \in C, \\
s_{i k}+t_{i}+t_{i j}-M\left(1-x_{i j k}\right) \leq s_{j k}, i, j \in N ; k \in V, \\
a_{i j k} \leq s_{i k} \leq b_{i}, i \in N ; k \in V, \\
\{0,1\}, y_{i j} \geq 0, z_{i j} \geq 0, i, j \in N, k \in V,
\end{gathered}
$$

The objective function (1) aims at minimizing total travel cost and the fixed cost of caregivers. Constraints (2) ensure that each patient is visited only once. Constraints (3) guarantee that a caregiver leave the patient after visiting this patient. Constraints (4)-(5) denote that every caregiver starts from the HHC depot, visits several patients and ends at the laboratory. Constraints (6) indicate that the total number of patients served by a caregiver cannot exceed the given constant. Constraints (7) describe the skill requirements assign strategy. Constraints (8)-(9) illustrate that the service cannot exceed the limitations of the time windows. Constraints (10) mean that decision variables are binary. 


\section{3. the travel and service times uncertainty}

As discussed in the introduction section, real-world HHC services are often subject to a variety of uncertainties. Therefore, a deterministic HHCRSP, which ignores the uncertainty in data, may not be an appropriate choice; while a robust optimization model, which does consider uncertainty, may be more suitable and reasonable for decision-making. Under the guidance of Robust optimization technique originally proposed by Ben-Tal \& Nemirovski (1999), in this work, we consider a robust version of the HHCRSP with both travel and service times uncertainty. To effectively represent the uncertain parameters, robust optimization requires a practical and clear definition of the uncertainty sets.

As we have mentioned before, our work mainly involves two kinds of uncertainties. Now, we define two types of uncertainties sets for $k$ th vehicle: namely the patient's service time uncertainty set $U_{s}^{k}$ and the travel time uncertainty set $U_{t}^{k}$ (Hu et al., 2018; Wu et al., 2017).

We assume that the travel time $\tilde{t_{i j}}$ on each arc $(i, j)$ belongs to an uncertainty set $U_{t}^{k}$, without additional distribution assumptions. Meanwhile, we assume the uncertain service time $\tilde{t_{i}}$ on each patient $i$ is with respect to the uncertainty set $U_{s}^{k}$.

$$
U_{s}^{k}=\left\{\tilde{t}_{i} \in R^{\left|N^{k}\right|}\left|\tilde{t}_{i}=\overline{t_{i}}+\alpha_{i} \hat{t}_{i}, \sum_{i \in N^{k}}\right| \alpha_{i}\left|\leq \Gamma_{s}^{k},\right| \alpha_{i} \mid \leq 1, \Gamma_{s}^{k}=\left[\theta_{s}\left|N^{k}\right|\right], \forall i \in N^{k}\right\}
$$

Formulation (11) describes the uncertainty set $\mathcal{U}_{s}=\times_{k \in K} U_{s}^{k}$, which illustrates the service time for $i$ th patient in route $k . N^{k}$ represents the set of patients in a route served by caregiver $k$ (sometimes we can also call it as route $k$ ). $\tilde{t}_{i}$ describes the uncertain service time for patient $i$, and $\overline{t_{i}}$ represents the nominal service time for patient $i . \alpha_{i}$ is an auxiliary variable. $\Gamma_{s}^{k}$ is a variable from the budget uncertainty, and its value controls the level of the service uncertainty. $\theta_{s} \in[0,1]$ is a coefficient of the service time uncertainty budget. $\left[\theta_{s}\left|N^{k}\right|\right]$ is the least integer value greater than the real number $\theta_{s}\left|N^{k}\right|$.

$$
U_{t}^{k}=\left\{\tilde{t}_{i j} \in R^{\left|A^{k}\right|}\left|\tilde{t}_{i j}=\overline{t_{i j}}+\beta_{i j} \hat{t}_{i j}, \sum_{(i, j) \in A^{k}}\right| \beta_{i j}\left|\leq \Gamma_{t}^{k},\right| \beta_{i j} \mid \leq 1, \Gamma_{t}^{k}=\left[\theta_{t}\left|A^{k}\right|\right], \forall(i, j) \in A^{k}\right\}
$$

Similar with equation (11), function 12 reveals the uncertainty set $\mathcal{U}_{t}=\times_{k \in K} U_{t}^{k}$, which illustrates the travel time for $i$ th patient in route $k . A^{k}$ represents the set of arcs in route $k . \tilde{t_{i j}}$ describes the uncertain travel time for patient $i$, and $\overline{t_{i j}}$ represents the nominal travel time between vertex 
$i$ and $j . \beta_{i}$ is an auxiliary variable. $\Gamma_{s}^{t}$ is a variable from the budget uncertainty, and its value controls the level of the service uncertainty. $\theta_{t} \in[0,1]$ is a coefficient of the travel time uncertainty budget. $\left[\theta_{t}\left|A^{k}\right|\right]$ is the least integer value greater than the real number $\theta_{t}\left|S^{k}\right|$.

\subsection{Robust optimization version}

Since the uncertainty of the travel and service times affects the feasibility of a solution, our robust optimization seeks to obtain a solution that can be feasible for any realization. However, complete protection from adverse realizations comes at the expense of a severe deterioration of the

objective function. Therefore, the uncertain parameter sets over which the worst cases are computed should be chosen to achieve a trade-off between performance and protection against uncertainty. (Chen et al., 2016a).

According to the proposed route-dependent uncertainty sets and the definition of robust optimization, we can extend the deterministic model into the robust optimization model by rewriting the objective function and constraints of time-window.

Robust HHC Routing model:

$$
\begin{gathered}
\min \sup _{s \in \operatorname{ext}\left(\mathcal{U}_{s}\right), t \in \operatorname{ext}\left(\mathcal{U}_{t}\right)} \sigma \cdot \sum_{k \in V} c f_{k} \sum_{j \in C} x_{0 j k}(s, t)+\sum_{k \in V} \sum_{i \in N} \sum_{j \in N} c_{i j} x_{i j k}(s, t) \\
\tilde{s}_{i k}(s, t)+t_{i}+t_{i j}-M\left(1-x_{i j k}\right) \leq \tilde{s}_{j k}(s, t), i, j \in N ; k \in V, s \in \operatorname{ext}\left(\mathcal{U}_{s}\right), t \in \operatorname{ext}\left(\mathcal{U}_{t}\right) \\
a_{i} \leq \tilde{s}_{i k}(s, t) \leq b_{i}, i \in N ; k \in V, s \in \operatorname{ext}\left(\mathcal{U}_{s}\right), t \in \operatorname{ext}\left(\mathcal{U}_{t}\right) .
\end{gathered}
$$

in which, $\operatorname{ext}\left(\mathcal{U}_{s}\right)$ and $\operatorname{ext}\left(\mathcal{U}_{t}\right)$ are sets that contain all the extreme points of sets $\mathcal{U}_{s}$ and $\mathcal{U}_{t}$, respectively. Objective function 13 present that the goal of the model is to minimize the total cost among the worst cases. Constraints $14-15$ is the time-window constraint with the uncertainties. 13 is a serious formulation which is from the original definition of the Robust Optimization.

\section{Calculating the largest possible arrival time}

Infeasible solutions are permitted in the evolution of searching the solutions, because, in tightly constrained problems is easily trapped into local optimal. Penalties are added to the objective 
function for violated constraints. As known, in a deterministic model, the delayed time, which is the gap between latest due time and the arrival time of the vehicles, can be used to calculate

In the deterministic model, the computing of the arrival time is quite simple. However, when it comes to the robust optimization version, this becomes much complicated. In this section, based on the theory of budget, we develop a method to calculate the largest possible arrival time, which is quite complicated.

For a given route $k \in V$, let $r_{k}$ represent the set of nodes in route $k$, and $n_{k}=\left|r_{k}\right|$ be the number of nodes in route $k . r_{k}$ can be described as $r_{k}=\left\{v_{0}=\operatorname{depot}, v_{1}, \ldots, v_{j}, \ldots v_{n_{k}}, v_{n_{k}+1}=\right.$ lab $\}$, in which $v_{j}$ represents the $j$ th patient in this route. According to the time window constraints (14), we must have $\widetilde{S}_{v_{j+1}}=\max \left(\widetilde{S}_{v_{j}}+\widetilde{t}_{v_{j} v_{j+1}}+\widetilde{t_{v_{j}}}, a_{v_{j+1}}\right)$.

Let $S^{k}\left(v_{i}, \Gamma_{s}^{k}, \Gamma_{t}^{k}\right)$ be the largest possible arrival time for $v_{i}$ with the parameters $\Gamma_{s}^{k}$ and $\Gamma_{t}^{k}$. 
Now, the largest possible arrival time can be written as a recursive function (16).

$S^{k}\left(v_{i}, \Gamma_{s}^{k}, \Gamma_{t}^{k}\right)= \begin{cases}0 & i=0, \\ \max \left(a_{v_{i}}, S_{v_{i-1}}+\bar{t}_{v_{i-1} v_{i}}+\overline{t_{v_{i-1}}}\right) & 1 \leq i \leq n, \Gamma_{t}^{k}=\Gamma_{s}^{k}=0 ; \\ \max \left(a_{v_{i}}, S^{k}\left(v_{i-1}, 0, \Gamma_{t}^{k}-1\right)+\bar{t}_{v_{i-1} v_{i}}+\hat{t}_{v_{i-1} v_{i}}\right. & 1 \leq i \leq n, \Gamma_{s}^{k}=0, \Gamma_{t}^{k}>0 ; \\ \left.+\overline{t_{v_{i-1}}}, S^{k}\left(v_{i-1}, 0, \Gamma_{t}^{k}\right)+\bar{t}_{v_{i-1} v_{i}}+\overline{t_{v_{i-1}}}\right) & 1 \leq i \leq n, \Gamma_{t}^{k}=0, \Gamma_{s}^{k}>0 ; \\ \max \left(a_{v_{i}}, S^{k}\left(v_{i-1}, \Gamma_{s}^{k}-1,0\right)+\bar{t}_{v_{i-1} v_{i}}+\hat{t}_{v_{i-1}}\right. & \\ \left.+\overline{t_{v_{i-1}}}, S^{k}\left(v_{i-1}, \Gamma_{s}^{k}, 0\right)+\bar{t}_{v_{i-1} v_{i}}+\overline{t_{v_{i-1}}}\right) & \\ \left.+\overline{t_{v_{i-1}}}+\hat{t}_{v_{i-1}}, S^{k}\left(v_{i-1}, \Gamma_{s}^{k}-1, \Gamma_{t}^{k}-1\right)+\Gamma_{t}^{k}\right)+\bar{t}_{v_{i-1}}+\hat{t}_{v_{i}}+\overline{t_{v_{i-1}}}, S^{k}\left(v_{i-1},\right. & \\ \left.\Gamma_{s}^{k}-1, \Gamma_{t}^{k}\right)+\bar{t}_{v_{i-1} v_{i}}+\overline{t_{v_{i-1}}}+\hat{t}_{v_{i-1}}, & 1 \leq i \leq n, \Gamma_{s}^{k}>0 \& \Gamma_{t}^{k}>0 ; \\ \left.S^{k}\left(v_{i-1}, \Gamma_{s}^{k}, \Gamma_{t}^{k}-1\right)+\bar{t}_{v_{i-1} v_{i}}+\hat{t}_{v_{i-1} v_{i}}+\overline{t_{v_{i-1}}}\right) & \Gamma_{s}^{k}>i, \\ S^{k}\left(v_{i}, \Gamma_{s}^{k}-1, \Gamma_{t}^{k}\right) & \Gamma_{t}^{k}>i, \\ S^{k}\left(v_{i}, \Gamma_{s}^{k}, \Gamma_{t}^{k}-1\right) & \text { otherwise } \\ -\infty & \end{cases}$

As mentioned in the beginning of this section, the objective function is transfered to the penalty-

based formulation. The objective function $f(R)$ in (17) is utilized to calculate the objective value of solution $R$. In (17), cost $(R)$ is computed by (1), and $\delta_{t}$ indicates the penalty factor for a violation of time window.

$$
f(R)=\operatorname{cost}(R)+\delta_{t} \sum_{k \in K} \sum_{i=1}^{\left|r_{k}\right|} \max \left(0, S^{k}\left(v_{i}, \Gamma_{s}^{k}, \Gamma_{t}^{k}\right)-b_{v_{i}}\right)
$$




\section{Proposed approaches}

According to the previous analysis, we found that the sub-problem of the deterministic model is

a vehicle routing problem. Because the vehicle routing problem is an NP-hard problem, there is no doubt that this problem is also an NP-hard problem. When the size of a instance becomes slightly larger, the exact algorithm and commercial software seem powerless to solve the model. Inspired from the previous related works (Eshtehadi et al., 2018; Braaten et al., 2017; Solano-Charris et al., 2015; Adulyasak \& Jaillet, 2015), we adopt heuristic methods to solve the proposed deterministic model and the robust model. To show the efficiency of the heuristics, we design three heuristics which are Simulated Annealing, Tabu Search, and Variable Neighborhood Search, to solve the proposed models.

For each proposed algorithm, we first illustrate the basic operators of the algorithms, and the detailed pseudo is described to illustrate the specific procedures.

\subsection{Simulated Annealing}

Simulating Annealing (SA) is a meta-heuristic which was first proposed by N. Metropolis in 1953. The basic idea for simulated annealing is based on the similarity between the annealing process of solid matter in physics and the general combinatorial optimization problem. The physical annealing process consists of three parts: heating process, isotherm process, and cooling process. Compared with the traditional Hill Climbing algorithm, SA has better ability to get rid of the local optimal solutions. So far, SA has been widely used in engineering, such as VLSI, production scheduling, control engineering, machine learning, neural network, signal processing, and et. al. In this chapter, SA is adapted to solve the proposed models. The detailed of the operators and procedures are described in this section.

\subsubsection{The representation of the solution}

In the heuristic algorithm, the representation structure of the solution has an important significance. In this study, we fully consider the characteristics of the problem. We use a commonly used integer to encode the routes. "0" represents the path of segmentation. The other integers correspond to patient's number. For example, the string "24189056730" could be represented in Fig.1. 


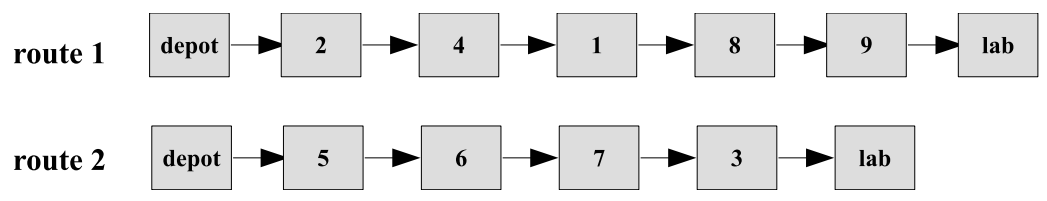

Figure 1: The representation a solution

\subsubsection{Initial solution}

The initial solution is obtained from a simple greedy algorithm. Firstly, the patients are simply divided into different groups according to their types of demand. Then, for each patient group with the requirement at level $i$, we employ the PFIH method, proposed by Solomon (1987), to get the initial route route_set_i. Finally, the initial solution for the whole problem is obtained just by merely combining the routes. The specific procedures for generating the initial solution are described in Figure 2 .

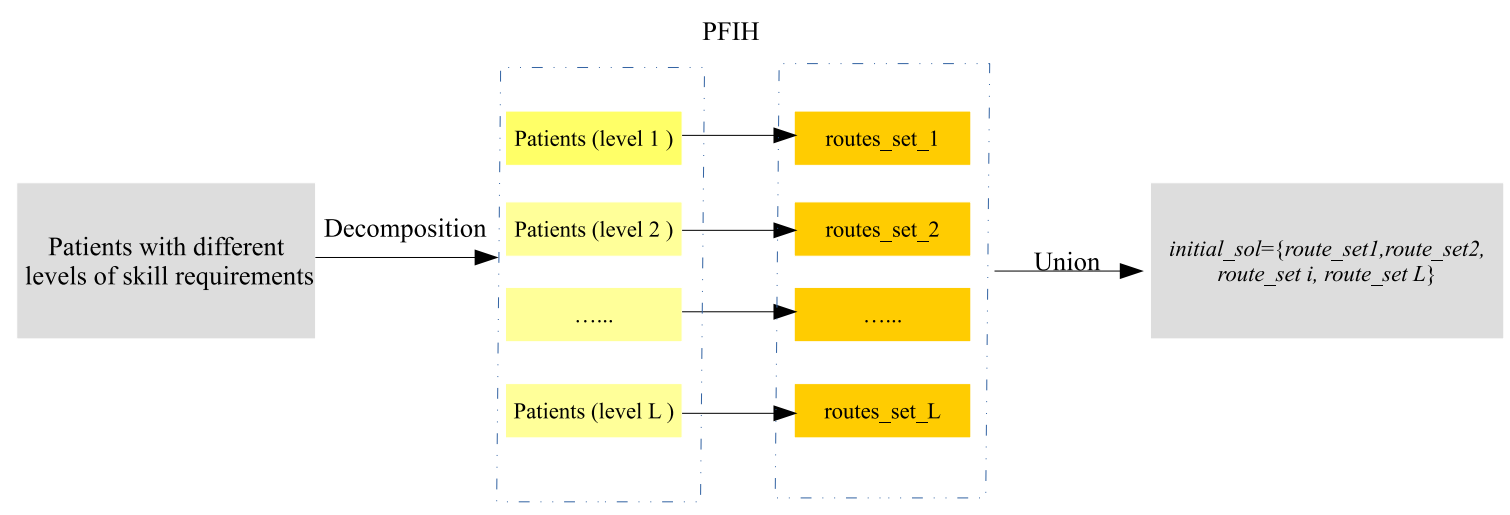

Figure 2: The procedure of generating initial solution

\subsubsection{Neighborhood structure}

The new solutions are generated from the neighborhood of the current solutions. In this work, six kinds of neighborhood structures are proposed. According to the behaviors of the nodes, we can classify the neighborhood into two categories, namely inter-route neighborhoods and intra-route neighborhoods. This section mainly depicts the procedures.

Three inter-route neighborhoods occur between two randomly selected routes.

inter-route insert: a vertex is randomly removed from a route, and then insert to another 
route.

inter-route swap: two vertices are randomly picked out from two routes respectively, then swap the two vertices.

inter-route 2-opt: two routes are broke down simultaneously, then reconnect to the other part from the breakpoints.

Three intra-route neighborhoods are only involved in one randomly selected singe route.

intra-route insert: a vertex is removed from the route and then insert to a different position of this route.

intra-route swap: two vertices are randomly picked out from the route and then exchange their positions.

intra-route reverse: two vertices are randomly selected as cutting points, then reverse the sequence of vertices between the two cutting points.

\subsubsection{Pseudo of $S A$}

In this section, we first introduce some frequently used parameters, and then present detailed procedures of the algorithm.

$365 T_{0}$ : the initial temperature.

$k$ : index of the iteration.

$T_{k}$ : the temperature in $k$ th iteration.

$s_{0}$ : initial solution.

$s^{\text {best }}$ : the global best solution obtained so far.

$\omega$ : the Boltzmann's Constant value.

$s^{\text {current }}$ : the current solution.

$s^{\text {new }}$ : the new solution obtained by a local search operator.

$r$ : a random value.

The main procedures for SA are shown in Algorithm 1. It starts from an initial solution $s_{0}$.

In the process of evolution, the neighborhood $\mathcal{N}(s)$ generates a random solution under a given temperature (see line 6). After that, in line 7, the gap between the objective values of a new solution and the current solution. In line 8 - line 12, the new solution replaces the new solution once the gap is less than 0 . Otherwise, in line 13 - line 17, the new solution is accepted in a probabilistic way. The global best solution is updated once the current solution is better than the 
current global best solution. The algorithm terminals once it reaches a temperature which is less than the given small number $\epsilon$, finally, the global best solution is output.

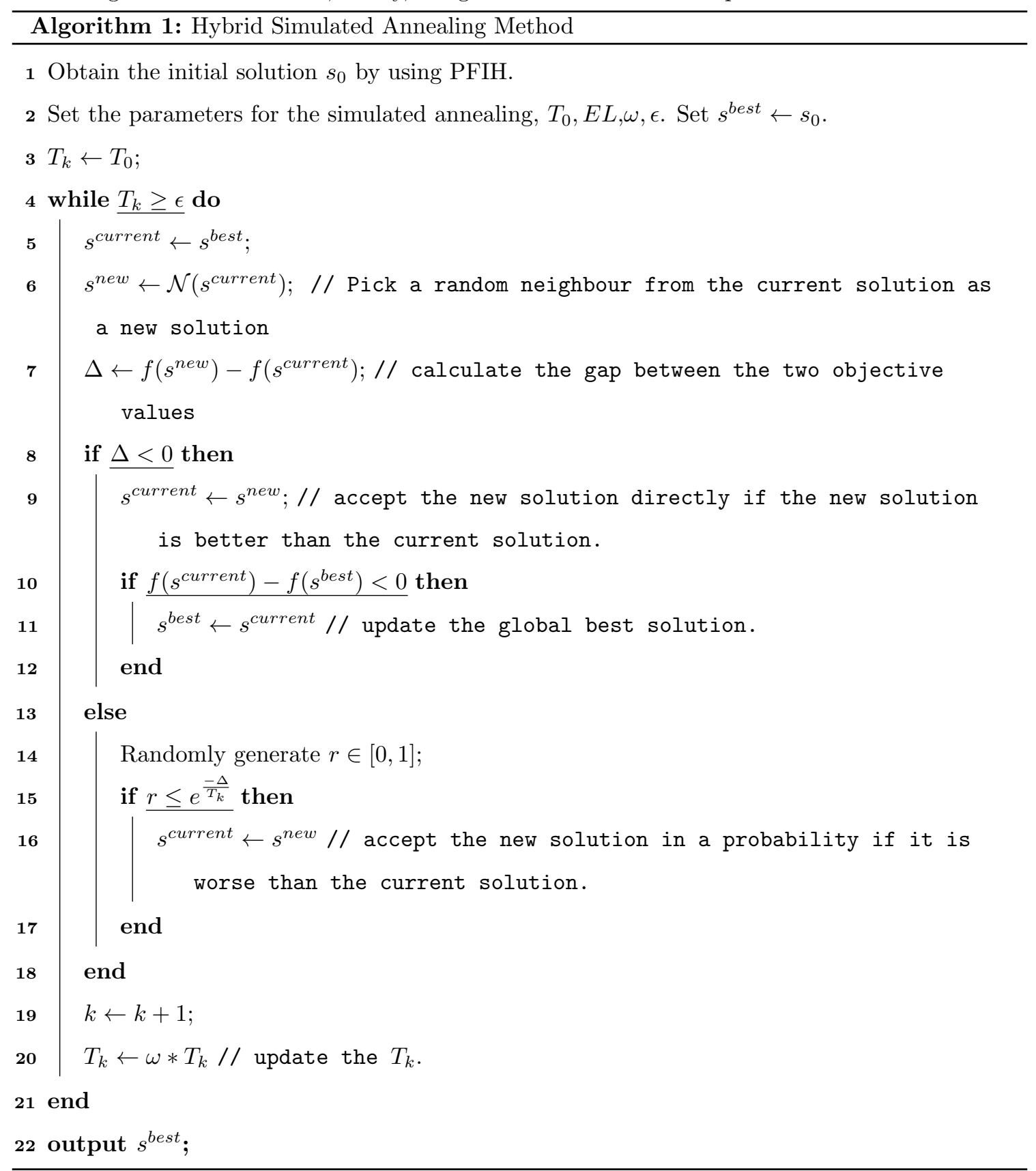




\subsection{Tabu Search}

Tabu Search algorithm is a meta-heuristic algorithm proposed by Glover in 1986. Starting from of solution. To avoid falling into the local optimal solution, TS uses a flexible "memory" technique to record and select the optimization process to guide the next search direction.

Tabu Search (TS) has been implemented for vast applications of combinatorial optimizations (Garcia et al., 1994; Taillard et al., 1997; Escobar et al., 2014; Silvestrin \& Ritt, 2017). In this section, we adapt the TS to solve the proposed model.

Our deterministic HHCRSP with skill-requirement can be viewed as a combination of VRPTW (Shi et al., 2017) and skill-assign problem. Time windows constraints, skill-assign are quite crucial constraints. The initial solution is obtained by utilizing the procedure 2 and the representation of a solution is the same with that used in the SA.

\subsubsection{Neighborhood}

As frequently used in many works (Wang et al., 2015; Silvestrin \& Ritt, 2017), $\lambda$-inter-exchange is a main local search operator applied in TS. The operator is conducted by interchanging patients between the routes. Each time, a pair of routes $\left(r_{p}, r_{q}\right)$ is selected, and the searching for the interchanging of patients is conducted sequentially. In this work, we consider the case $\lambda=2$; this indicates that at most two patients will be interchanged between each pair of routes. Generally speaking, the operations of 2-interexchange could be described as: $(0,1),(0,2),(1,1),(1,2),(2,1),(2,2)$. Let us explain the operator by giving an example. The operator $(1,2)$ on a route pair $\left(r_{p}, r_{q}\right)$ illustrates that, 2 patients will be shifted from $r_{q}$ to $r_{p}$, and 1 patient will be shifted from $r_{p}$ to $r_{q}$. The others are defined similarly. Only feasible solutions are considered in the process of generating new solutions.

\subsubsection{The structure of tabu list}

Let us consider that there are $n$ patients, $k$ vehicles, and the tabu list $T L$ is designed by a $n \times k$ matrix which is shown in Figure 3. The value of $T L(i, j)$ indicates that inserting patient $i$ to vehicle $j$ is forbidden in the next $\max (T L(i, j), 0)$ times. Anyone who is interested in this kinds of tabu list can refer to Silvestrin \& Ritt (2017)

Now, Let us give an example to explain how the tabu list works in our algorithm. In Figure $3, T L(1,1)=-5 \leq 0$ indicates that move patient 1 to a route served by caregiver 1 (also can be 
marked as route 1 for short.) is allowed in the following iteration; however, $T L(3,2)=3$ reveals that the moving patient 3 to a route 2 is impossible unless the aspiration encounters. This type of tabu list is quite simple, understandable but can represent all the movement of the inter-route. Besides, this structure of the tabu list is also convenient for updating the value of the table.

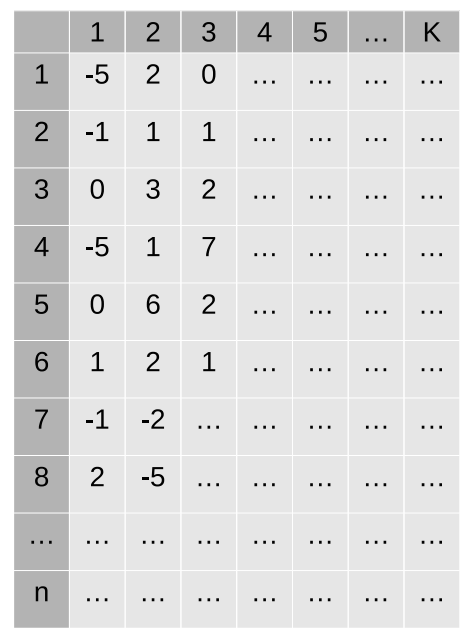

Figure 3: The array of the tabu list

\begin{tabular}{|c|c|c|c|c|c|c|c|}
\hline & 1 & 2 & 3 & 4 & 5 & $\ldots$ & $\mathrm{K}$ \\
\hline 1 & 1 & 2 & 0 & $\ldots$ & $\ldots$ & $\ldots$ & $\ldots$ \\
\hline 2 & 2 & 1 & 1 & $\ldots$ & $\ldots$ & $\ldots$ & $\ldots$ \\
\hline 3 & 3 & 3 & 2 & $\ldots$ & $\ldots$ & $\ldots$ & $\ldots$ \\
\hline 4 & 1 & 2 & 4 & $\ldots$ & $\ldots$ & $\ldots$ & $\ldots$ \\
\hline 5 & 2 & 1 & 2 & $\ldots$ & $\ldots$ & $\ldots$ & $\ldots$ \\
\hline 6 & 6 & 2 & 1 & $\ldots$ & $\ldots$ & $\ldots$ & $\ldots$ \\
\hline 7 & 1 & 1 & $\ldots$ & $\ldots$ & $\ldots$ & $\ldots$ & $\ldots$ \\
\hline 8 & 0 & 2 & $\ldots$ & $\ldots$ & $\ldots$ & $\ldots$ & $\ldots$ \\
\hline$\ldots$ & $\ldots$ & $\ldots$ & $\ldots$ & $\ldots$ & $\ldots$ & $\ldots$ & $\ldots$ \\
\hline $\mathrm{n}$ & $\ldots$ & $\ldots$ & $\ldots$ & $\ldots$ & $\ldots$ & $\ldots$ & $\ldots$ \\
\hline
\end{tabular}

Figure 4: The array of the frequency list

\subsubsection{Intensification and Diversification}

The traditional simple TS is also easily trapped into the local optimal solutions. To overcome this issue, Glover (1986) proposed two senior operators which are intensification and diversification, 
to benefit searching better solution. In this TS, we utilize the frequency-based intensification and diversification operators to guide the searching. The frequency list is established for recording the frequency of the used moves. Figure 4 describes a frequency list $F L . F L(i, j)=p$ indicates that the movement of inserting patient $i$ to vehicle $j$ has occurred for $p$ times.

Intensification strategies are based on the modification of choice rules to encourage the historically good combinations of motion and solution features. They can also initiate a return to attractive regions to search for them in more depth. In our research, for each specific iteration, we try to move to the highest-frequency direction.

Diversification is a primary operator to guide the search into new regions. Frequency-based diversification is realized by penalizing the high frequency of used moves.

Let $s$ be a solution candidate generated from $s^{\text {current }}$. Set $P$ represents the available moves excluding the forbidden ones. $p^{\prime}$ indicates the moves from $s^{\text {current }}$ to $s . g\left(s^{\text {current }}, p^{\prime}\right)$ is used to calculate the frequency of the movement from $F L . \lambda$ is the value of current iterations. $\alpha \in[0,1]$ is a random number. Consider that the current solution is a local optimum whose objective value is $f$. We choose the best move according to the modified objective function $f^{\prime}$ from equation (18).

$$
f^{\prime}(s)=f(s)\left(1+\alpha \sum_{p^{\prime} \in P} g\left(s^{\text {current }}, p^{\prime}\right) / \lambda\right),
$$

\subsubsection{Aspiration criterion and stopping criterion}

The aspiration criterion overrides the tabu status of a move $\left(r^{\prime}, r\right)$ if this move yields a solution $s^{\prime}$ such that $f\left(s^{\prime}\right)$ is better than the global best solution found so far. We prefer to accept this movement no matter whether it should be forbidden because this situation would not like to cause global best solution in $\mu$ iterations. 


\subsubsection{The pseudo code of TS}

The detailed description of TS is depicted in Algorithm 2. Our TS starts from the initial solution $s_{0}$, which is obtained based on the procedures in the Figure 2. After that, in line 2, we initialize the best solution and current solution as $s_{0}$, and we set the tabu list and the frequency list as an empty matrix. Line 3-18 gives the detailed procedures for improving the best solution $s^{\star}$. The tabu list and the frequency list are updated after each iteration. And the TS terminates when it reaches the stopping criterion. Finally, the algorithm returns to the best solution and the current solution.

Algorithm 2: The pseudo code of TS

1 Input: initial solution $s_{0}$;

2 Initialize Current best solution $s^{\star} \leftarrow s_{0}$, current solution $s^{\text {current }} \leftarrow s_{0}$. Initialize tabu list

$T L$, frequency list $F L$, tabu length $L$, and the parameter of terminal criterion.

3 while It does not reach the stopping criterion do

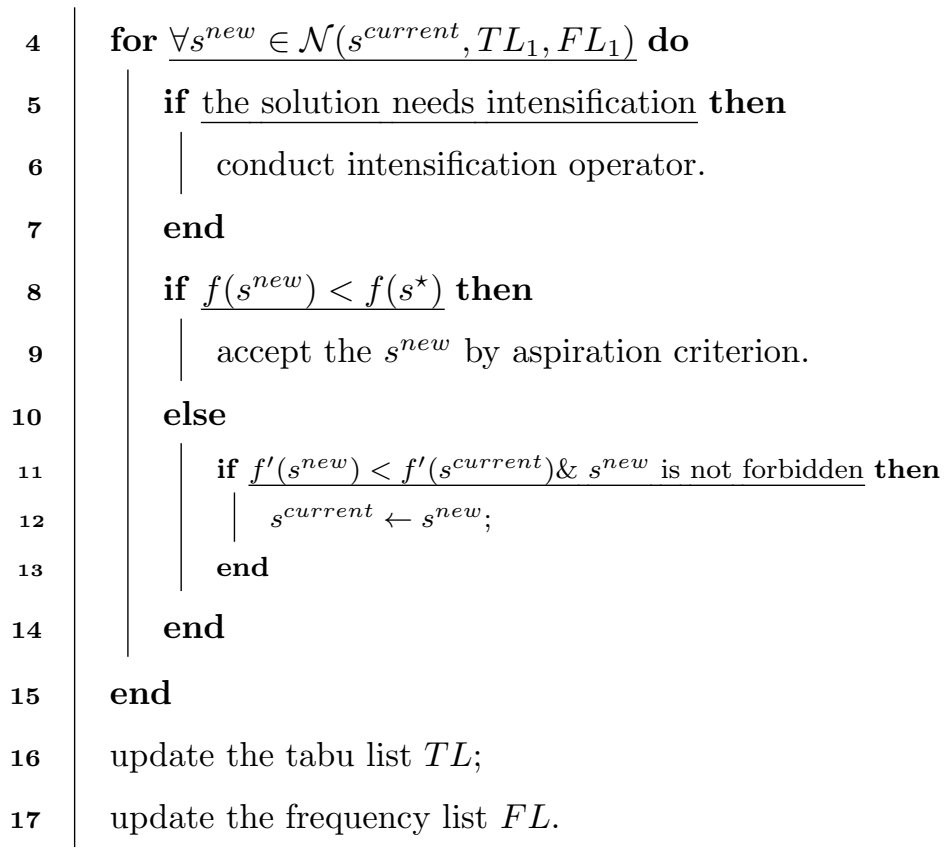

18 end

19 Return: $s \star$ and $s^{\text {current }}$.

\subsection{Variable Neighborhood Search (VNS)}

The Variable Neighborhood Research (VNS) is a classical meta-heuristic which is proposed by Mladenović, Hansen, 1997. It is usually applied to solve a set of combinatorial optimization 
problems (Bortfeldt et al., 2015; Sarasola et al., 2016). The main idea of the variable neighborhood search algorithm is to apply multiple different neighborhoods for the process of searching. First, 455 the smallest neighborhood is employed to obtain a better solution. When the solution cannot be improved, switch to a slightly larger neighborhood. If it can continue to improve the solution, return to the smallest neighborhood, otherwise continue to switch to a larger neighborhood.

The detailed procedures of VNS is given in Algorithm 3. Our VNS also starts from the initial solution $s_{0}$. In line 2 , we initialize the best solution as $s_{0}$, and set the parameters for the algorithm. 460 Lines 3-26 give the detailed procedures for improving the best solution $s^{\star}$. The procedure in line 7 illustrates the switching of neighborhood structure. And, lines 9-17 show the strategy of generating new solutions. VNS terminates when it reaches the stopping criterion: the best solution has non- 
improvement for certain iterations. Finally, the algorithm returns the best solution.

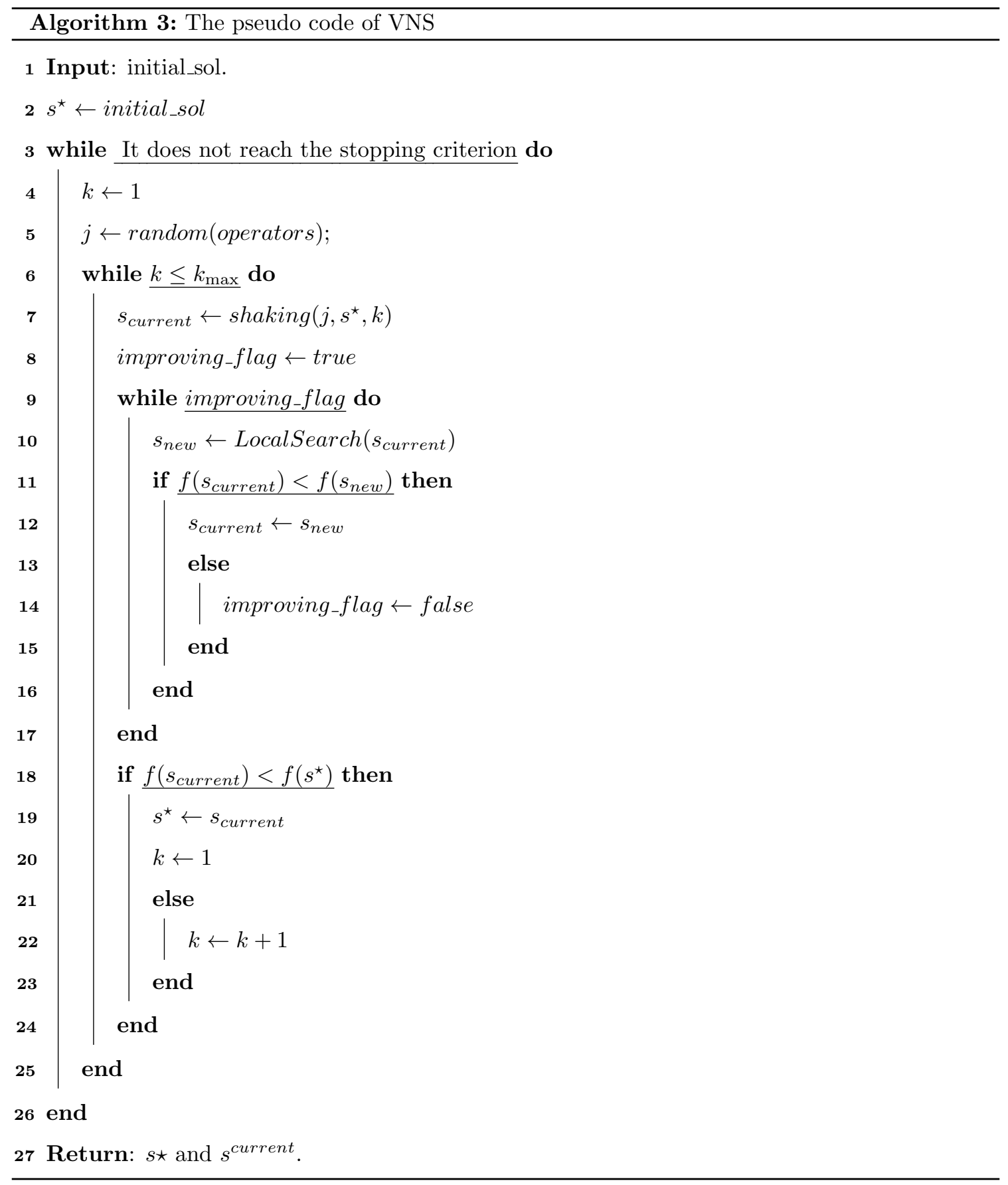




\section{Experiments}

To validate the proposed models and algorithms, we have performed several series of experiments. In this section, firstly, corresponding instances are introduced in section 6.1 , then section 6.2 reports the experimental results of the deterministic model which is solved by Gurobi Solver, SA, VNS, TS respectively. After that, the detailed experiments and analysis of robust model are presented in section 6.3. Finally, the comparison between the solutions obtained by the robust optimization model and stochastic programming with recourse is performed. All the heuristic algorithms are implemented in Java.

\section{1. introduction to the instances}

To the best of our knowledge, there is no standard benchmark in the literature for our problem. the first $\eta$ of the patients are corresponding to the first level service, while the rest patients are corresponding to the second level service. For example, when $\eta=0.6$, and the size of the instance is 
50 patients, then we define the first 30 patients with the first level service and the other 20 patients with second level service.

(3) The close time of the patient's time window is regarded as the latest starting service time.

(4) The maximum number of patients which can be serviced by a caregiver is 10 .

The six groups of instances have featured by the distribution of the location and length of time window. The data source are suitable for our problem for two reasons.

(1) In the HHC service, the location of patients has different features. For example, in some area, patients come from some communities; consequently, the locations are more likes cluster type (C1 and C2 instances). While, sometimes, the services are ordered randomly; therefore, the location of the patients is distributed randomly, so, this scenario corresponds to random type (R1 and R2 instances). More commonly, the distribution of patients' location is mixed with random and cluster, which is RC type (RC1 and RC2).

(2) In the HHC service, a different type of patients may prefer a different length of time windows. For example, some patients can be serviced in a long period during the day, this situation can be corresponding to the width time window $(\mathrm{R} 1, \mathrm{C} 1, \mathrm{RC} 1)$, while some patients tend to provide service just in a short time, which is a narrow time window ( $22, \mathrm{C} 2, \mathrm{RC} 2)$.

To sum up, the generated instances almost cover all the possible scenarios of location distribution and time window in $\mathrm{HHC}$ service.

To distinguish the classic instances of Solomon, we name them RO-XXX. The main parameters used in the proposed algorithms are listed in Table 2 and explained below. All the values of them are empirically chosen. 
Table 2: Parameters used in the model algorithms

\begin{tabular}{|c|c|c|}
\hline Model \& Algorithms & Parameters & Values \\
\hline \multirow{5}{*}{ Model } & $\sigma$ : the weight between employee fees and travel cost. & 1 \\
\hline & $\eta$ : the percentage to describe the skill requirement of patients & 0.6 \\
\hline & $f c_{1}$ : fixed cost for caregiver with skills in level 1 & 50 \\
\hline & $f c_{2}$ : fixed cost for caregiver with skills in level 2 & 80 \\
\hline & $\delta_{t}$ : the big data to weight penalty and total cost of schedule $R$ & 20000 \\
\hline \multirow{5}{*}{$\mathrm{SA}$} & $T_{0}$ : the initial temperature. & 100 \\
\hline & $E L:$ epoch length for local search in a certain temperature & 50 \\
\hline & $M L$ : the max iterations for accepting new feasible solutions in a certain temperature. & 80 \\
\hline & $\epsilon$ : the threshold of temperature for stopping algorithm. & 0.1 \\
\hline & $\omega:$ the Boltzmann's Constant value. & 0.9995 \\
\hline VNS & $\mu$ : the max iteration for non-improvement (terminal conditions) & 100 \\
\hline \multirow{3}{*}{ TS } & $\alpha:$ the parameter for adjusting the diversification operator & 0.1 \\
\hline & tabu_length: the length for the tabu list & a random integer in $[5,10]$ \\
\hline & the max iteration for non-improvement (terminal conditions) & 100 \\
\hline
\end{tabular}
other two methods. 
Table 3: The experimental results for solutions obtained by Gurobi Solver

\begin{tabular}{c|cccccc}
\hline \multirow{2}{*}{ ID } & \multicolumn{7}{|c}{ GUROBI SOLVER } \\
\cline { 2 - 7 } & NV_level1 & NV_level2 & TC_upper & TC_lower & CT(s) & gap1\% \\
\hline RO-RCdp0501 & 1 & 2 & 428.82 & 428.82 & 0.22 & $0.00 \%$ \\
RO-RCdp0504 & 1 & 1 & 335.54 & 278.00 & $7,200.00$ & $20.70 \%$ \\
RO-RCdp0507 & 0 & 2 & 384.34 & 258.03 & $7,200.00$ & $48.95 \%$ \\
RO-RCdp1001 & 2 & 2 & 633.63 & 633.63 & 0.02 & $0.00 \%$ \\
RO-RCdp1004 & 1 & 1 & 346.85 & 320.84 & $7,200.00$ & $8.11 \%$ \\
RO-RCdp1007 & 2 & 1 & 476.92 & 365.55 & $7,200.00$ & $30.47 \%$ \\
\hline
\end{tabular}

Table 4: Experimental results obtained by TS, VNS and SA for solving small-size instances

\begin{tabular}{l|cccc|cccccc|ccccc}
\hline & \multicolumn{5}{|c}{ TS } & \multicolumn{5}{c|}{ SA } & \multicolumn{5}{c|}{ VNS } \\
\hline ID & N_level1 & N_level2 & TD & TC & N_level1 & N_level2 & TD & TC & gap1 & N_level1 & N_level2 & TD & TC & gap2 \\
\hline RO-Rcdp0501 & 1 & 2 & 218.82 & 428.82 & 1 & 2 & 218.82 & 428.82 & $0.00 \%$ & 1 & 2 & 218.82 & 428.82 & $0.00 \%$ \\
RO-Rcdp0504 & 1 & 1 & 205.54 & 335.54 & 1 & 1 & 205.54 & 335.54 & $0.00 \%$ & 1 & 1 & 205.54 & 335.54 & $0.00 \%$ \\
RO-Rcdp0507 & 1 & 1 & 224.34 & 354.34 & 0 & 2 & 224.34 & 384.34 & $8.47 \%$ & 0 & 2 & 224.34 & 384.34 & $8.47 \%$ \\
RO-Rcdp1001 & 1 & 2 & 375.95 & 585.95 & 1 & 2 & 383.63 & 593.63 & $1.31 \%$ & 1 & 2 & 383.63 & 593.63 & $1.31 \%$ \\
RO-Rcdp1004 & 1 & 1 & 216.85 & 346.85 & 1 & 1 & 222.80 & 352.80 & $1.72 \%$ & 1 & 1 & 216.85 & 346.85 & $0.00 \%$ \\
RO-Rcdp1007 & 2 & 1 & 266.92 & 446.92 & 1 & 2 & 266.92 & 476.92 & $6.71 \%$ & 1 & 2 & 266.92 & 476.92 & $6.71 \%$ \\
RO-Rcdp2501 & 2 & 3 & 617.81 & 957.81 & 2 & 3 & 679.21 & $1,019.21$ & $6.41 \%$ & 3 & 3 & 718.04 & $1,108.04$ & $15.68 \%$ \\
RO-Rcdp2504 & 3 & 2 & 540.99 & 850.99 & 3 & 2 & 610.45 & 920.45 & $8.16 \%$ & 3 & 2 & 598.81 & 908.81 & $6.79 \%$ \\
RO-Rcdp2507 & 2 & 3 & 577.61 & 917.61 & 2 & 3 & 613.62 & 953.62 & $3.92 \%$ & 2 & 3 & 582.61 & 922.61 & $0.54 \%$ \\
RO-Rcdp5001 & 5 & 5 & $1,064.67$ & $1,714.67$ & 5 & 6 & $1,084.38$ & $1,814.38$ & $5.82 \%$ & 5 & 5 & $1,109.20$ & $1,759.20$ & $2.60 \%$ \\
RO-Rcdp5004 & 5 & 3 & 870.75 & $1,360.75$ & 5 & 3 & 940.28 & $1,430.28$ & $5.11 \%$ & 5 & 3 & 885.54 & $1,375.54$ & $1.09 \%$ \\
RO-Rcdp5007 & 5 & 4 & 905.84 & $1,475.84$ & 4 & 5 & $1,059.13$ & $1,659.13$ & $12.42 \%$ & 5 & 4 & $1,002.20$ & $1,572.20$ & $6.53 \%$ \\
\hline
\end{tabular}

\subsubsection{Comparison between the heuristic methods for 25-patient instances}

In this section, the experimental results of the proposed three heuristics (SA, TS, VNS) for 25-patient instances are presented. In table 5, the first column is the ID of the instances, then column 2-5, 6-11, and 13-17 illustrate the results of TS, SA, VNS respectively. For each presented results, we give the number of the assigned caregivers in level 1 (N_level1) and level 2 (N_level2), the total transportation distance (TD), and total cost (TC) which is weighted by travel distance and employee fees of caregivers. Other two heuristics are compared with TS. To analyze the results, we define four parameters named gap11, gap12, gap 21, and gap22 respectively. Gap1 shows the difference of TC between TS and SA. Gap12 computes the gap of the number of used caregivers between TS and SA. Evidently, gap2 and gap21 have the similar meaning with gap11 and gap12 respectively. As shown in table $5, \mathrm{SA}$ and VNS can get almost the same objective values, but TS can get better results than the others. 
Table 5: The experimental results obtained by TS, SA, and VNS for solving the deterministic model with 25-patient

\begin{tabular}{|c|c|c|c|c|c|c|c|c|c|c|c|c|c|c|c|c|}
\hline \multirow{2}{*}{ ID } & \multicolumn{4}{|c|}{ TS } & \multicolumn{6}{|c|}{$\mathrm{SA}$} & \multicolumn{6}{|c|}{ VNS } \\
\hline & N_level1 & N_level2 & $\mathrm{TD}$ & TC & N_level1 & N_level2 & $\mathrm{TD}$ & $\mathrm{TC}$ & gap1 & gap11 & N_level1 & N_level2 & TD & TC & gap2 & gap21 \\
\hline RO-C101 & 1 & 2 & 257.3114 & 467.3114 & 1 & 2 & 271.8507 & 481.8507 & $3.11 \%$ & 0 & 1 & 2 & 259.811 & 469.811 & $0.53 \%$ & 0 \\
\hline RO-C102 & 1 & 2 & 270.1773 & 480.1773 & 1 & 2 & 258.0508 & 468.0508 & $-2.53 \%$ & 0 & 1 & 2 & 254.938 & 464.938 & $-3.17 \%$ & 0 \\
\hline RO-C103 & 2 & 1 & 271.4741 & 451.4741 & 1 & 2 & 280.1854 & 490.1854 & $8.57 \%$ & 0 & 2 & 2 & 325.8666 & 585.8666 & $29.77 \%$ & 1 \\
\hline RO-C104 & 2 & 1 & 275.4859 & 455.4859 & 1 & 2 & 288.5523 & 498.5523 & $9.46 \%$ & 0 & 2 & 1 & 302.6885 & 482.6885 & $5.97 \%$ & 0 \\
\hline RO-C105 & 1 & 2 & 257.3114 & 467.3114 & 1 & 2 & 273.0785 & 483.0785 & $3.37 \%$ & 0 & 1 & 2 & 259.216 & 469.216 & $0.41 \%$ & 0 \\
\hline RO-C106 & 1 & 2 & 257.3114 & 467.3114 & 1 & 2 & 271.8507 & 481.8507 & $3.11 \%$ & 0 & 1 & 2 & 259.216 & 469.216 & $0.41 \%$ & 0 \\
\hline RO-C107 & 1 & 2 & 259.811 & 469.811 & 1 & 2 & 285.3323 & 495.3323 & $5.43 \%$ & 0 & 1 & 2 & 259.216 & 469.216 & $-0.13 \%$ & 0 \\
\hline RO-C108 & 1 & 2 & 270.4514 & 480.4514 & 1 & 2 & 274.5631 & 484.5631 & $0.86 \%$ & 0 & 1 & 2 & 257.5717 & 467.5717 & $-2.68 \%$ & 0 \\
\hline RO-C109 & 1 & 2 & 251.6258 & 461.6258 & 1 & 2 & 280.4307 & 490.4307 & $6.24 \%$ & 0 & 1 & 2 & 256.8845 & 466.8845 & $1.14 \%$ & 0 \\
\hline RO-R101 & 3 & 5 & 646.6733 & 1196.6733 & 2 & 6 & 658.4145 & 1238.4145 & $3.49 \%$ & 0 & 3 & 5 & 684.2169 & 1234.2169 & $3.14 \%$ & 0 \\
\hline RO-R102 & 4 & 3 & 591.9535 & 1031.9535 & 3 & 4 & 623.0914 & 1093.0914 & $5.92 \%$ & 0 & 3 & 4 & 616.74 & 1086.74 & $5.31 \%$ & 0 \\
\hline RO-R103 & 2 & 3 & 459.9161 & 799.9161 & 1 & 4 & 543.8293 & 913.8293 & $14.24 \%$ & 0 & 1 & 4 & 491.6077 & 861.6077 & $7.71 \%$ & 0 \\
\hline RO-R104 & 1 & 3 & 430.1738 & 720.1738 & 1 & 3 & 507.3581 & 797.3581 & $10.72 \%$ & 0 & 2 & 3 & 498.3211 & 838.3211 & $16.41 \%$ & 1 \\
\hline RO-R105 & 3 & 3 & 537.2049 & 927.2049 & 2 & 4 & 575.7211 & 995.7211 & $7.39 \%$ & 0 & 2 & 4 & 575.7211 & 995.7211 & $7.39 \%$ & 0 \\
\hline RO-R106 & 2 & 3 & 496.0873 & 836.0873 & 1 & 4 & 616.4634 & 986.4634 & $17.99 \%$ & 0 & 1 & 4 & 518.2423 & 888.2423 & $6.24 \%$ & 0 \\
\hline RO-R107 & 2 & 2 & 433.7158 & 693.7158 & 2 & 2 & 484.757 & 744.757 & $7.36 \%$ & 0 & 3 & 2 & 544.8937 & 854.8937 & $23.23 \%$ & 1 \\
\hline RO-R108 & 2 & 2 & 414.153 & 674.153 & 1 & 3 & 476.1161 & 766.1161 & $13.64 \%$ & 0 & 2 & 2 & 490.0817 & 750.0817 & $11.26 \%$ & 0 \\
\hline RO-R109 & 3 & 2 & 464.6926 & 774.6926 & 1 & 4 & 527.0552 & 897.0552 & $15.79 \%$ & 0 & 2 & 4 & 530.386 & 950.386 & $22.68 \%$ & 1 \\
\hline RO-R110 & 2 & 3 & 452.7869 & 792.7869 & 1 & 4 & 482.4932 & 852.4932 & $7.53 \%$ & 0 & 2 & 3 & 485.8331 & 825.8331 & $4.17 \%$ & 0 \\
\hline RO-R111 & 3 & 2 & 465.224 & 775.224 & 1 & 4 & 504.0917 & 874.0917 & $12.75 \%$ & 0 & 2 & 3 & 510.2525 & 850.2525 & $9.68 \%$ & 0 \\
\hline RO-R112 & 2 & 2 & 421.7127 & 681.7127 & 0 & 4 & 453.2384 & 773.2384 & $13.43 \%$ & 0 & 1 & 3 & 515.3154 & 805.3154 & $18.13 \%$ & 0 \\
\hline RO-RC101 & 2 & 3 & 427.1824 & 767.1824 & 2 & 3 & 441.093 & 781.093 & $1.81 \%$ & 0 & 2 & 3 & 427.1824 & 767.1824 & $0.00 \%$ & 0 \\
\hline RO-RC102 & 1 & 2 & 342.7479 & 552.7479 & 1 & 3 & 481.3234 & 771.3234 & $39.54 \%$ & 1 & 2 & 3 & 408.7155 & 748.7155 & $35.45 \%$ & 2 \\
\hline RO-RC103 & 2 & 2 & 352.2787 & 612.2787 & 1 & 2 & 358.6876 & 568.6876 & $-7.12 \%$ & -1 & 1 & 2 & 320.7717 & 530.7717 & $-13.31 \%$ & -1 \\
\hline RO-RC104 & 2 & 2 & 322.2515 & 582.2515 & 1 & 2 & 354.6025 & 564.6025 & $-3.03 \%$ & -1 & 2 & 2 & 325.8324 & 585.8324 & $0.62 \%$ & 0 \\
\hline RO-RC105 & 3 & 2 & 454.5723 & 764.5723 & 2 & 2 & 417.8423 & 677.8423 & $-11.34 \%$ & -1 & 2 & 2 & 376.6754 & 636.6754 & $-16.73 \%$ & -1 \\
\hline RO-RC106 & 1 & 2 & 334.7958 & 544.7958 & 2 & 2 & 375.3318 & 635.3318 & $16.62 \%$ & 1 & 1 & 2 & 334.7958 & 544.7958 & $0.00 \%$ & 0 \\
\hline RO-RC107 & 2 & 1 & 290.2782 & 470.2782 & 1 & 2 & 339.9725 & 549.9725 & $16.95 \%$ & 0 & 1 & 2 & 289.5183 & 499.5183 & $6.22 \%$ & 0 \\
\hline RO-RC108 & 1 & 2 & 283.5855 & 493.5855 & 1 & 2 & 338.4584 & 548.4584 & $11.12 \%$ & 0 & 1 & 2 & 279.722 & 489.722 & $-0.78 \%$ & 0 \\
\hline RO-C201 & 2 & 1 & 337.6001 & 517.6001 & 1 & 2 & 332.8254 & 542.8254 & $4.87 \%$ & 0 & 2 & 1 & 357.8473 & 537.8473 & $3.91 \%$ & 0 \\
\hline RO-C202 & 2 & 1 & 328.0293 & 508.0293 & 1 & 2 & 341.7611 & 551.7611 & $8.61 \%$ & 0 & 2 & 1 & 354.6819 & 534.6819 & $5.25 \%$ & 0 \\
\hline Avg. & & & & & & & & & $7.93 \%$ & -0.03 & & & & & $6.07 \%$ & 0.13 \\
\hline
\end{tabular}

\subsection{Robust analysis for the instances}

In the last section, the deterministic model is solved by the proposed three heuristics. Furthermore, in this section, the robust model is dealt with the proposed heuristics. What's more, after obtaining the solutions, we also analysis that how the location distribution of patients, the length of time-windows, the uncertainty of time-windows, and the uncertainty of service times affect the robust solutions.

As we have emphasized, a perfect schedule for an HHC company should not only have less operational cost but also robust for the service. To evaluate the obtained solutions from the perspective of robustness, we introduce some indicators to measure the character of each schedule.

Firstly, $V_{0}, V_{1}, V_{2}, V_{3}$ are proposed to measure the number of delayed services.

${ }_{555} V_{0}$ : the probability of serving all patients timely. 
$V_{1}$ : the probability of not serving one patient timely at most

$V_{2}$ : the probability of not serving two patients timely at most

$V_{3}$ : the probability of not serving three patients timely at most.

To further describe the service level, $M E T, D P S$, and $M D T$ are introduced to get the statistic results from Monte Carlo Simulation.

$M E T$ : mean extra working times for each caregiver.

$D P S$ : the percentage of the delayed service for patients.

$M D T$ : mean delayed time for each patient.

To measure the robustness of each solution, we perform a Monte Carlo simulation to calculate the value of the proposed indicators. In the simulation, we assume that the travel and service times are uncertain but subject to a limited range. The maximum travel time gap for $t_{i j}$ is $0.2 * t_{i j}$, and the gap of maximum service time $t_{i}$ for each patient $i$ is $0.2 * t_{i}$. The uncertainty budget coefficient $\theta_{t} \in[0,1]$ determines the uncertainty budget of $\Gamma_{t}^{k}$. The simulating procedures can be viewed in Figure 5 .

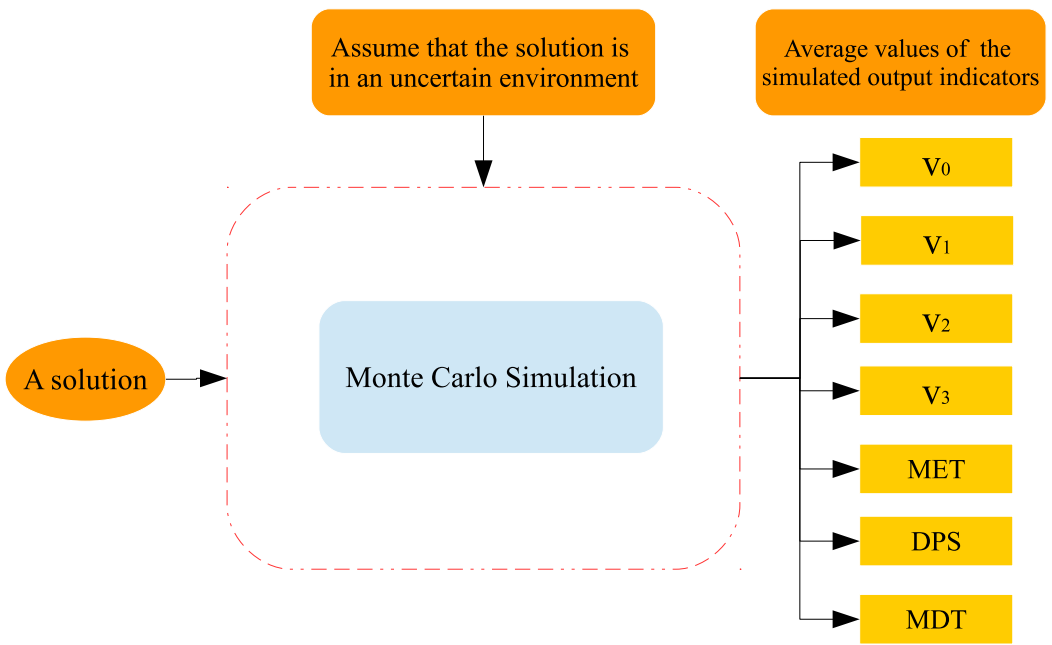

Figure 5: Simulating procedure for evaluating each solution 


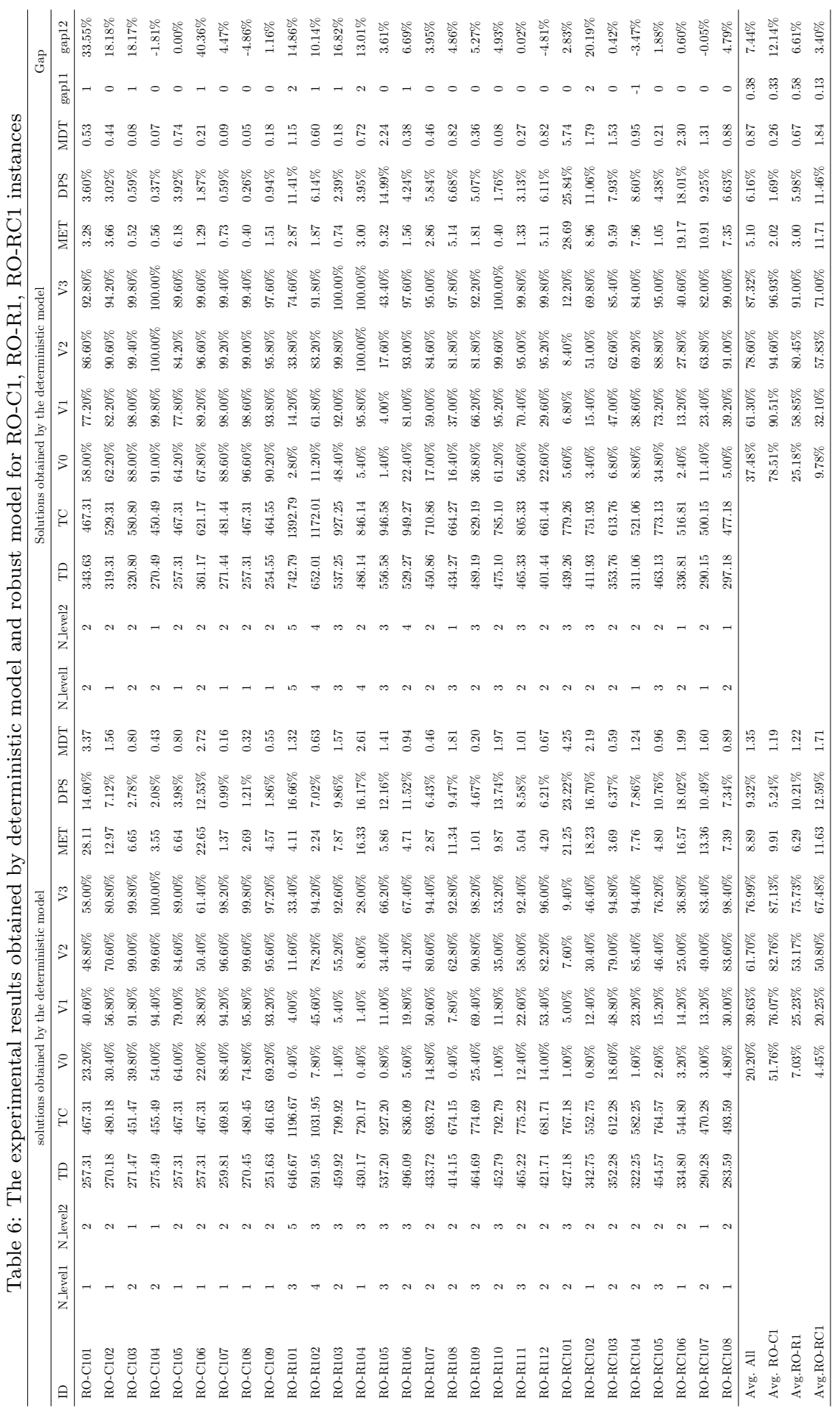




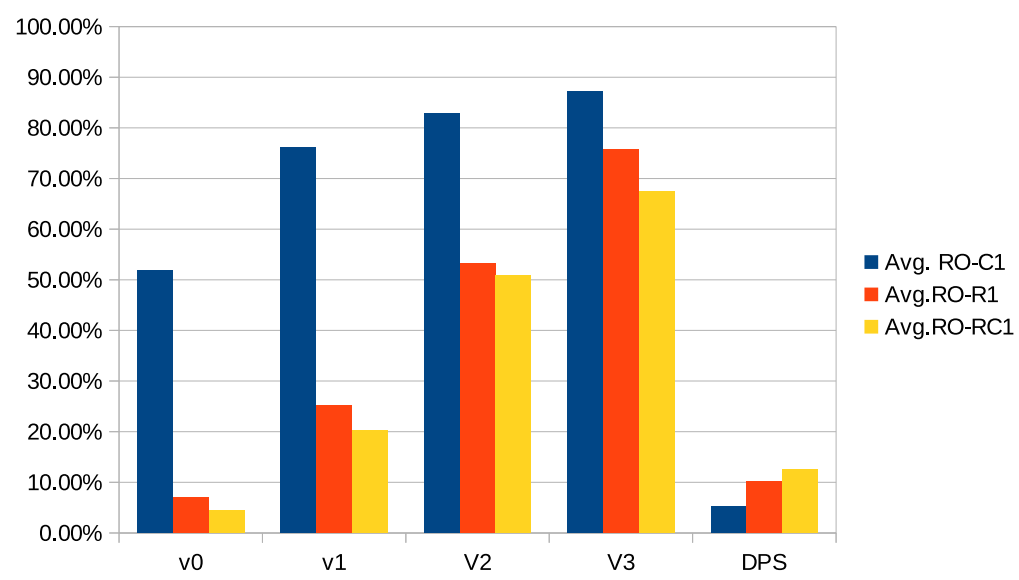

Figure 6: Solutions obtained by deterministic model for instances RO-C1, RO-R1, and RO-RC1 with 25 patients

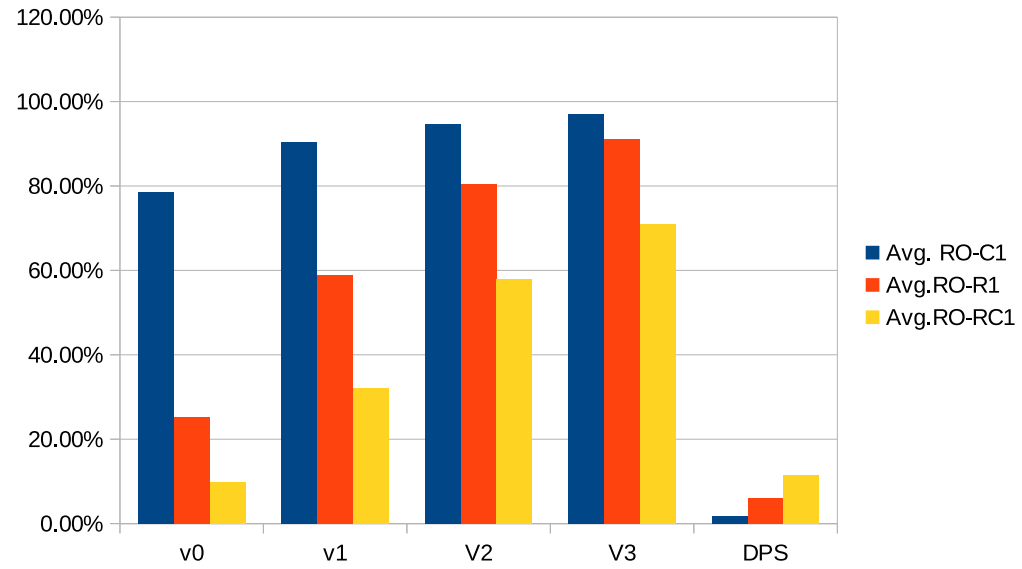

Figure 7: Solutions obtained by robust model for instances RO-C1, RO-R1, and RO-RC1 with 25 patients

As discussed in section 6.1, instances in different datasets have different types of patients' locations. To show the effect of different types of locations, we present the details of deterministic and robust solutions for all instances of datasets RO-R1, RO-RC1 and RO-C1 together in Table 6. In these tables, the columns under "Deterministic" display the details of each deterministic solution.

The columns under "Robust" show the details of each robust solution. The columns named "gap" show the increase in the number of vehicles used and the percentage increase in the total distance traveled by the robust solution compared to the deterministic solution in each case. Note that only 
the results of the simulation tests taking into account both the uncertainty of the travel and service time were included. A detailed analysis of the effect of patient location models leads to conclusions

580 similar to those above.

As shown in Figure 6 and 7, the deterministic solutions for the instances in the three data sets are very fragile, in particular, the solutions for the instances $\mathrm{R} 1$ and $\mathrm{RC} 1$. For example, the average probability of serving each client was only $7.03 \%$ with deterministic solutions for R1 instances and $4.45 \%$ with deterministic solutions for RC1 instances, while this value becomes $51.76 \%$ with the $\mathrm{RO}-\mathrm{C} 1$

However, in robust solutions, the average number of vehicles used for RO-R1 instances increased by 0.38 , and the average total distance traveled increased by $7.44 \%$ over deterministic solutions.

Thus, even if the robust solution significantly reduces the risk of non-compliance with the client period, decision makers still have to evaluate the trade-off between total distance traveled and the robustness of the road in situations with clustered location models.

6.3.2. The Effect of the length of time window 


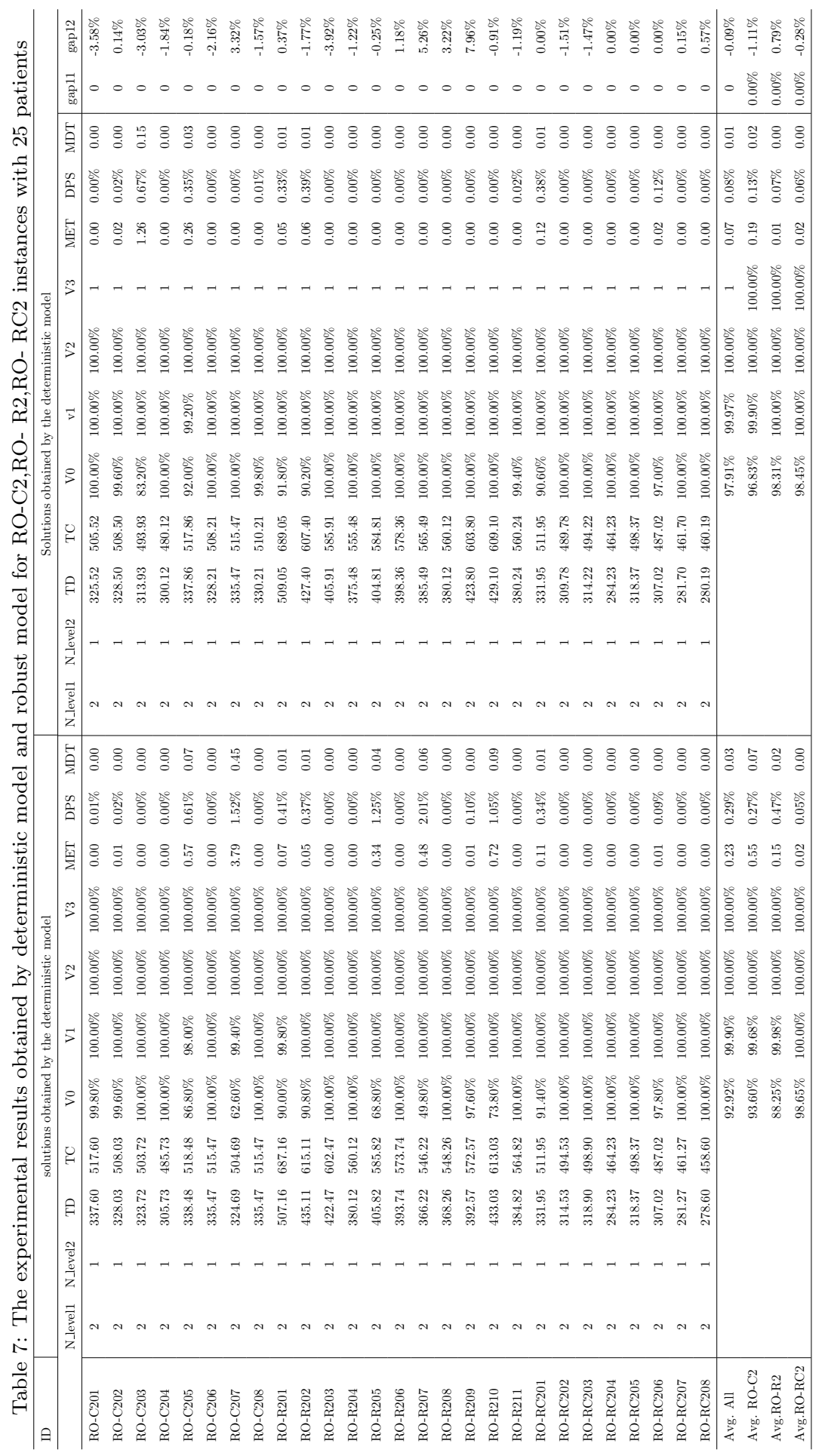




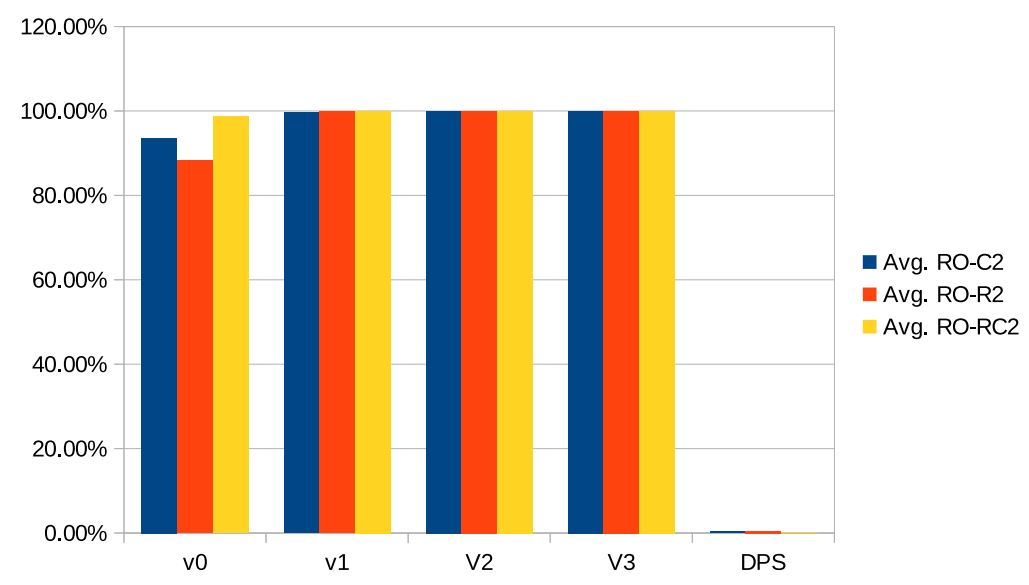

Figure 8: Solutions obtained by deterministic model for instances RO-C2, RO-R2, and RO-RC2 with 25 patients

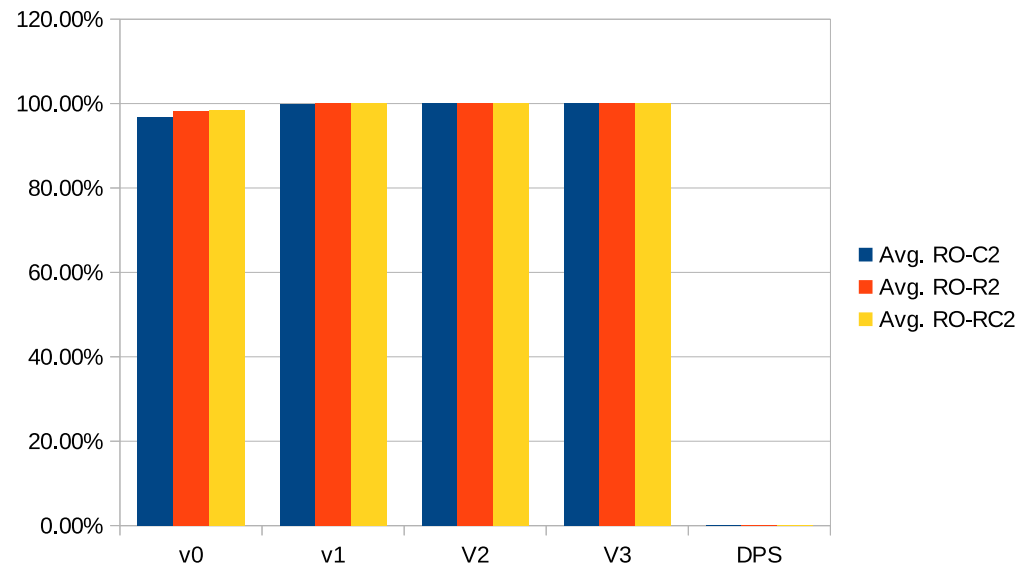

Figure 9: Solutions obtained by robust model for instances RO-C2, RO-R2, and RO-RC2 with 25 patients

Unlike the instances of RO-R1, RO-C1, and RO-RC1, the instances of RO-R2, RO-RC2, and RO-C2 have a long planning horizon for each patient. These features serve many patients along the same route. To show the effect of wide time windows on planned routes, we present the details of deterministic and robust solutions for RO- R2, RO-RC2 and RO-C2 instances in Table 7.

Based on the results in Figures 8 and 9, it is clear that the deterministic solutions for the RO$\mathrm{R} 2, \mathrm{RO}-\mathrm{RC} 2$ and $\mathrm{RO}-\mathrm{C} 2$ instances are more robust than the deterministic solutions for the RO-R1, RO-RC1, and RO-C1 instances. 
For example, the average probability of serving each patient with deterministic solutions for ROa comparison between the solutions obtained by the RO model and stochastic programming with recourse.

\subsection{1. comparison between deterministic model and $R O$}

Furthermore, we have also solved the generated instances with 50 and 100 patients, respectively. Cinstances was $88.25 \%$, indicating that these deterministic solutions are not very fragile. In robust solutions for RO-R2, RO-RC2 and RO-C2 instances, the total travel distance increased slightly, and the number of vehicles used remained the same in most cases. However, the robustness of the routes with these solutions has improved considerably. For example, the average probability of serving all patients was $96.83 \%, 98.31 \%$, and $98.45 \%$ for RO-R2, RO-RC2, and RO-C2, respectively. These results indicate that the solutions are very robust given travel time and uncertainty of service time.

We can, therefore, conclude that a high level of route robustness can be achieved in cases where large windows use robust solutions at almost zero cost. However, not serving all patients is more prevalent in deterministic solutions, in cases where time windows are narrow, and ensuring a high level of robustness of roads is much more expensive, as shown in Tables 6 .

\subsection{Comparison with other models}

To highlight the proposed RO model, we perform three series of comparison. First, we make a comparison between the RO with the deterministic model, which does not take into account uncertainties. Then, the comparison among the following four models: the deterministic model, RO model with considering travel and service times uncertainties, RO model with only consider travel time uncertainty, and RO model with only consider service time uncertainty. Finally, we give his section mainly evaluates and analyzes these solutions by comparing them with the deterministic model. For convenience, we call the deterministic model as Deter. model for short. The detailed experimental results are illustrated in Appendix Table B.13, B.14, B.15, and B.16. In order to explain the simulation results, we draw Figure 10 to 13.

Figure 10, which is composed of four sub-figures, describes the simulation results of the indicators of Vs. Let us explain by giving an example of sub-figure 1. The x-axis is the identification number of instances, and in this figure, we have 56 instances totally. Meanwhile, the y-axis denotes the value of V0. We can notice that, in almost all cases, the solutions obtained by RO shows a higher value than the solution obtained by the Deter. model. This scenario explains that the solutions 
generated by RO have less delayed service than that produced by Deter. model. Other indicators also show the same tendency as sub-figure 1.
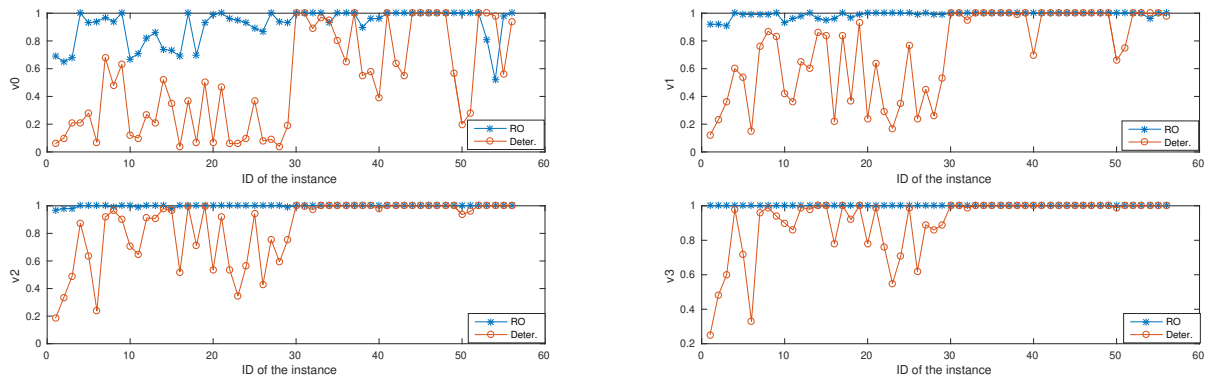

Figure 10: The indicators of $\mathrm{V}_{i}$ obtained by $\mathrm{RO}$ model and deterministic model instances with 50 patients

Figure 11 depicts the other three meaningful indicators, MET, DPS, and MDT, which represent the overall delay service. For example, in the first sub-figure, the X-axis also illustrates the ID of 56 instances, while Y-axis indicates the MET (Mean Extra Time). We find that, in almost all the cases, the solutions obtained by RO tend to have less mean extra time than that achieved by Deter. model. Therefore, we also summarize that the solutions obtained by RO show more advantage in Percentage of Delayed Service (DPS) and Mean Delayed Time (MDT) than that generated by deterministic model..
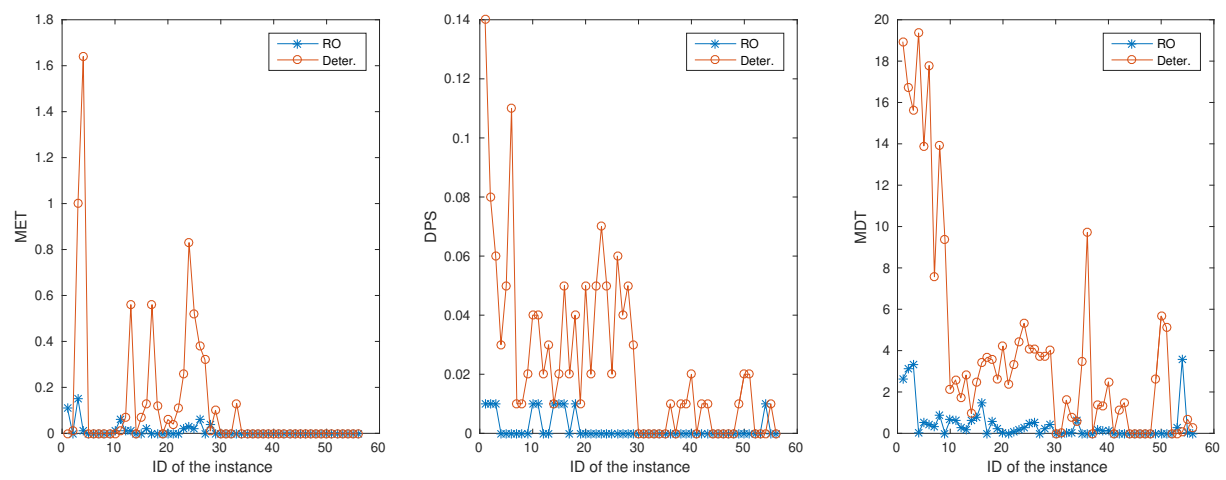

Figure 11: The indicators of MET, DPS, and MDT obtained by RO model and deterministic model for instances with 50 patients 

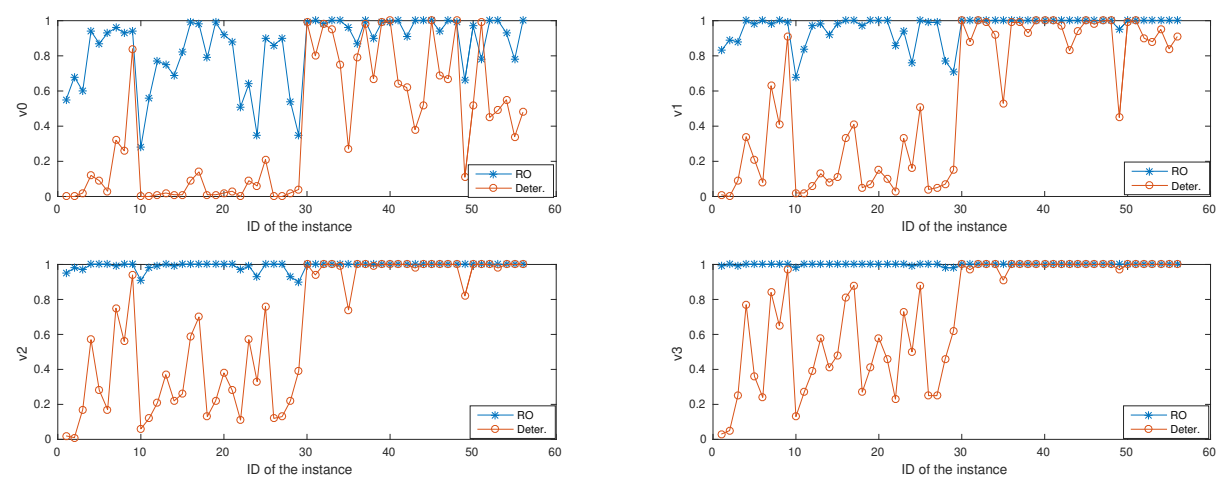

Figure 12: The indicators of $\mathrm{V}_{i}$ obtained by $\mathrm{RO}$ model and deterministic model instances with 100 patients
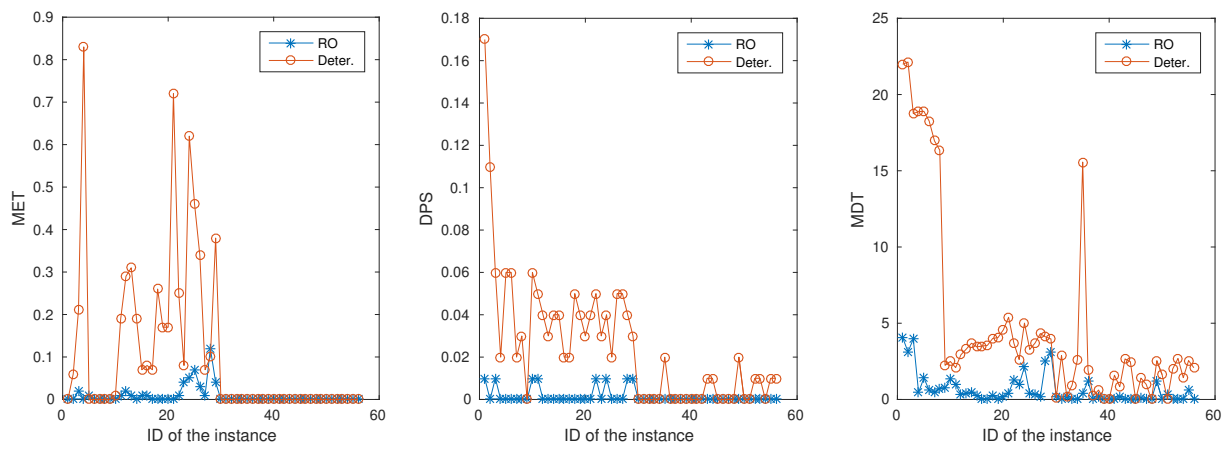

Figure 13: The indicators of MET, DPS, and MDT obtained by RO model and deterministic model for instances with 100 patients

The simulation indicators for the 100-patient instances are shown in Figure 13 and 12. We can find that the figures also show the same tendency as that of 11 and 10 respectively.

\subsection{2. comparison between deterministic model, US, UT, and USUT}

To show the influence of the uncertain travel and service times individually, we define four models DM, US, UT, USUT as follows.

Deter. model: the model with deterministic travel and service times (namely the deterministic model). 
US model: the model with only consideration of uncertain service time.

UT model: the model with only consideration of uncertain travel time.

USUT model: the model with taking into account uncertain service and travel time.

As shown in Table 8, the average simulation results of the solutions obtained by the four models are presented. For RO-C1, the percentage of being able to service all patients timely is $51.76 \%$, ${ }_{650}$ and this value becomes $63.36 \%$ and $72.07 \%$ respectively once considering either uncertain travel or service times. However, the percentage increases to $78.51 \%$ if we consider both the uncertain travel and service times simultaneously. Other types of instances have the similar trends. This trend reveals that the solutions obtained by USUT have strong robustness than that achieved by US, and UT, while the solutions obtained by DM shows the worst robustness. 
Table 8: Comparison among the solutions obtained by the four models for the instances with 25 patients

\begin{tabular}{l|lccccccc}
\hline Model & & V0 & V1 & V2 & V3 & MET & DPS & MDT \\
\hline \multirow{2}{*}{ Deter. Model } & Avg. RO-C1 & $51.76 \%$ & $76.07 \%$ & $82.76 \%$ & $87.13 \%$ & 9.91 & $5.24 \%$ & 1.19 \\
& Avg. RO-R1 & $7.03 \%$ & $25.23 \%$ & $53.17 \%$ & $75.73 \%$ & 6.29 & $10.21 \%$ & 1.22 \\
& Avg. RO-RC1 & $4.45 \%$ & $20.25 \%$ & $50.80 \%$ & $67.48 \%$ & 11.63 & $12.59 \%$ & 1.71 \\
& Avg. RO-C2 & $93.60 \%$ & $99.68 \%$ & $100.00 \%$ & $100.00 \%$ & 0.55 & $0.27 \%$ & 0.07 \\
& Avg. RO-R2 & $88.25 \%$ & $99.98 \%$ & $100.00 \%$ & $100.00 \%$ & 0.15 & $0.47 \%$ & 0.02 \\
& Avg. RO-RC2 & $98.65 \%$ & $100.00 \%$ & $100.00 \%$ & $100.00 \%$ & 0.02 & $0.05 \%$ & 0 \\
\hline \multirow{5}{*}{ US model } & Avg. RO-C1 & $63.36 \%$ & $80.49 \%$ & $86.76 \%$ & $90.76 \%$ & 6.58 & $3.84 \%$ & 0.81 \\
& Avg. RO-R1 & $13.33 \%$ & $32.83 \%$ & $58.15 \%$ & $79.18 \%$ & 4.43 & $9.10 \%$ & 0.91 \\
& Avg. RO-RC1 & $12.73 \%$ & $39.10 \%$ & $56.33 \%$ & $68.55 \%$ & 11.92 & $11.68 \%$ & 1.84 \\
& Avg. RO-C2 & $97.80 \%$ & $99.83 \%$ & $99.93 \%$ & $100.00 \%$ & 0.16 & $0.10 \%$ & 0.02 \\
& Avg. RO-R2 & $91.95 \%$ & $99.98 \%$ & $100.00 \%$ & $100.00 \%$ & 0.11 & $0.32 \%$ & 0.01 \\
& Avg. RO-RC2 & $98.80 \%$ & $100.00 \%$ & $100.00 \%$ & $100.00 \%$ & 0.02 & $0.05 \%$ & 0.00 \\
\hline \multirow{5}{*}{ US model } & Avg. RO-C1 & $72.07 \%$ & $84.38 \%$ & $91.58 \%$ & $95.07 \%$ & 3.75 & $2.53 \%$ & 0.48 \\
& Avg. RO-R1 & $16.18 \%$ & $39.42 \%$ & $66.70 \%$ & $84.28 \%$ & 3.94 & $8.05 \%$ & 0.83 \\
& Avg. RO-RC1 & $6.08 \%$ & $21.83 \%$ & $39.70 \%$ & $58.75 \%$ & 15.81 & $13.35 \%$ & 2.42 \\
& Avg. RO-C2 & $99.20 \%$ & $99.88 \%$ & $100.00 \%$ & $100.00 \%$ & 0.03 & $0.04 \%$ & 0.00 \\
& Avg. RO-R2 & $94.13 \%$ & $99.13 \%$ & $99.98 \%$ & $100.00 \%$ & 0.11 & $0.27 \%$ & 0.01 \\
& Avg. RO-RC2 & $98.45 \%$ & $100.00 \%$ & $100.00 \%$ & $100.00 \%$ & 0.03 & $0.06 \%$ & 0.00 \\
\hline Avg. RO-C1 & $78.51 \%$ & $90.51 \%$ & $94.60 \%$ & $96.93 \%$ & 2.02 & $1.69 \%$ & 0.26 \\
& Avg. RO-R1 & $25.18 \%$ & $58.85 \%$ & $80.45 \%$ & $91.00 \%$ & 3.00 & $5.98 \%$ & 0.67 \\
& Avg. RO-RC1 & $9.78 \%$ & $32.10 \%$ & $57.83 \%$ & $71.00 \%$ & 11.71 & $11.46 \%$ & 1.84 \\
& Avg. RO-C2 & $96.83 \%$ & $99.90 \%$ & $100.00 \%$ & $100.00 \%$ & 0.19 & $0.13 \%$ & 0.02 \\
& Avg. RO-R2 & $98.31 \%$ & $100.00 \%$ & $100.00 \%$ & $100.00 \%$ & 0.01 & $0.07 \%$ & 0 \\
& Avg. RO-RC2 & $98.45 \%$ & $100.00 \%$ & $100.00 \%$ & $100.00 \%$ & 0.02 & $0.06 \%$ & 0 \\
\hline
\end{tabular}

As mentioned in the literature part, Stochastic Programming with Recourse (SPR) is another critical framework for modeling optimization problem when dealing with uncertainties. In this section, we attempt to make a comparison between the solutions obtained by the RO model and SPR model. Notably, we refer to the SPR models investigated by Shi et al. (2018); Li et al. (2010). Shi et al. (2018) investigated an HHC routing and scheduling problem with considering uncertain travel and service times, while, Li et al. (2010) deal with the stochastic travel and service time in VRPTW. The detailed formulations of SPR model for HHCRSP can be found in Appendix A. Meanwhile, the solution and simulation results are listed in Tables B.11 and B.12, which are located 
in the Appendix B.

As analyzed before, the instances RO-C2, RO-R2, RO-RC2 characterized with long timewindows, show strong robustness regardless of considering uncertain travel and service times or not. According to Table B.12, these instances also illustrate strong robustness, which is quite reasonable. Therefore, we care more about the instances with RO-C1, RO-R1 and RO-RC1, who characterized narrow time-window. Because, these instances always meet with challenges of robustness when ignoring uncertainties. The detailed analysis of the experimental results in Table B.11 will be performed in this section.

To depict the experimental results in Table B.11 more clear, we obtain Figure 14 and 15 from tables in Appendix B.
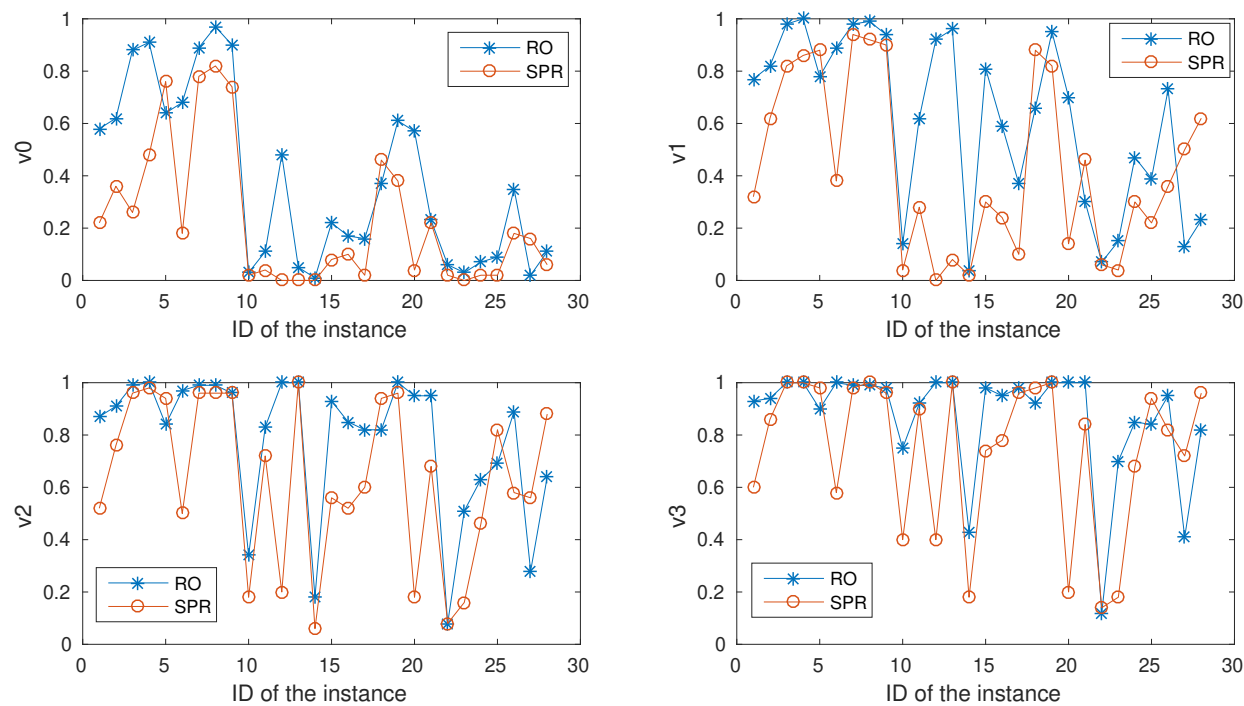

Figure 14: The indicators of $\mathrm{V}_{i}$ obtained by RO model and SPR model for RO-R1, RO-C1, RO-RC1 type instances with 25 patients.

As shown in Figure 14, there are totally four sub-figures, each of which describes the simulation results of the indicators of Vs. For example, in sub-figure 1, the x-axis is the identification number of instances. Meanwhile, the y-axis represents the value of V0. As we find that, in almost all the cases, the solutions obtained by RO (the blue line with a start on it) shows a higher value than the 
solution obtained by SPR (orange line with a circle on it). This situation reveals that the solutions generated by RO show less delayed service than that produced by SPR. If we analogize to subgraph 1, we can get a similar conclusion.
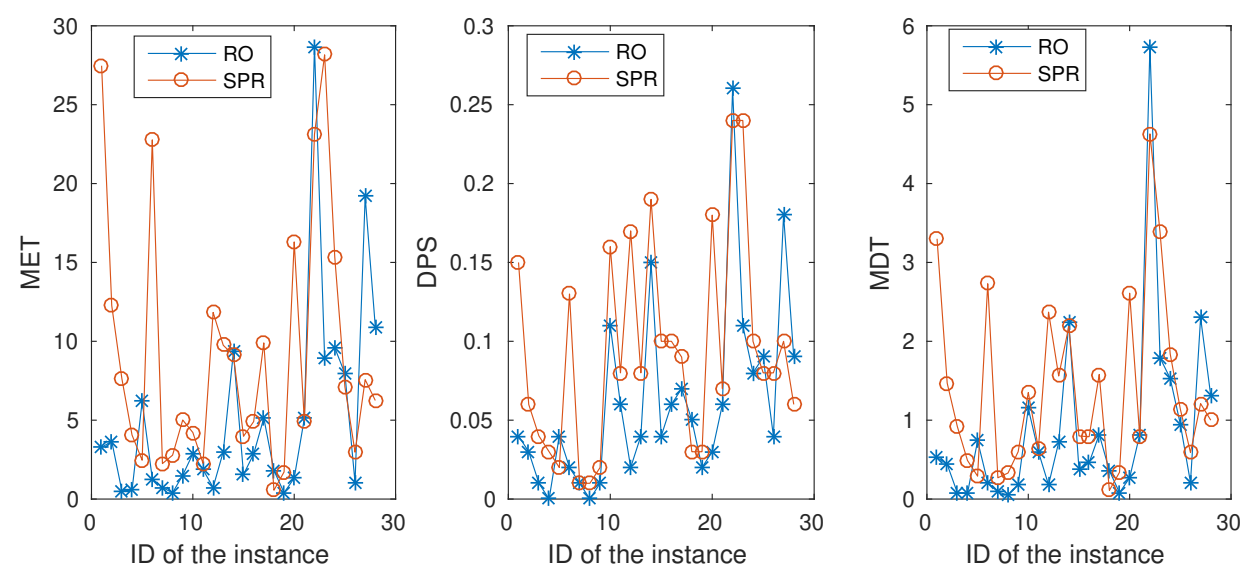

Figure 15: The indicators of MET, DPS, and MDT obtained by RO model and SPR model for RO-R1, RO-C1, RO-RC1 type instances with 25 patients.

Figure 15 shows another three significant indicators, MET, DPS, and MDT, which describe the overall delay service situation. For example, in the first sub-figure, the $\mathrm{X}$-axis also illustrates the ID of instances, while Y-axis indicates the MET (Mean Extra Time). We find that, in almost all the cases, the solutions obtained by RO tend to have less mean extra time than that achieved by SPR. Therefore, we also summarize that the solutions obtained by RO show more advantage in Percentage of Delayed Service (DPS) and Mean Delayed Time (MDT) than that generated by SPR.

To further demonstrate the comparison between the solutions obtained by RO model and SPR model from the statistic perspective, we have performed a Friedman-test, which is a non-parametric statistical test, initially proposed by Friedman (1940). This statistic test intends to detect differences in treatments across multiple test attempts. A lot of researchers, such as Derrac et al. (2011); Zafra et al. (2011); Osaba et al. (2016, 2019); Shi et al. (2018) has successfully applied Friedmantest to effectively identify different approaches or models. Here, we target to identify two different methods, which, of course, are RO model and SPR model. 
As a paradigm, hypothesis are always designed before carrying out statistic test. Here, two hypothesis are described as follows.

$H_{0}$ : The difference between the results obtained by the two models follows a symmetric distribution around zero.

$H_{1}$ : the difference between the results achieved by the two models does not follow a symmetric distribution around zero.

We have performed Friedman-tests on indicators achieved by two models respectively. The pvalues are reported in Table 6.5. In the table, the average ranking located in the third row shows the numerical value rankings between the solutions obtained by a model (either RO or SPR) for each indicator. Here we should emphasize that V0, V1, V2, V3 are positive indicators to show robustness. These indicators have the meaning that the higher the ranking, the stronger robustness will be. On the contrary, MET, DPS, and MDT are negative values, which indicates that the lower the ranking, the stronger robustness will have. For each solution, the ranking will be either 1 or 2 , in which, rank 1 is senior to rank 2 just from the numerical value.

Table 9: The results of the Friedman-tests for the solutions obtained by the RO model and SPR model

\begin{tabular}{|c|c|c|c|c|c|c|c|c|c|c|c|c|c|c|}
\hline indicators & \multicolumn{2}{|c|}{ V0 } & \multicolumn{2}{|c|}{ v1 } & \multicolumn{2}{|c|}{$\mathrm{v} 2$} & \multicolumn{2}{|c|}{ v3 } & \multicolumn{2}{|c|}{ MET } & \multicolumn{2}{|c|}{ DPS } & \multicolumn{2}{|c|}{ MDT } \\
\hline models & $\mathrm{RO}$ & SPR & $\mathrm{RO}$ & SPR & $\mathrm{RO}$ & SPR & $\mathrm{RO}$ & SPR & $\mathrm{RO}$ & SPR & $\mathrm{RO}$ & SPR & $\mathrm{RO}$ & SPR \\
\hline Avg. ranking & 1.11 & 1.89 & 1.18 & 1.82 & 1.18 & 1.82 & 1.25 & 1.75 & 1.71 & 1.29 & 1.75 & 1.25 & 1.75 & 1.25 \\
\hline P-values & \multicolumn{2}{|c|}{0.0002} & \multicolumn{2}{|c|}{0.0019} & \multicolumn{2}{|c|}{0.0010} & \multicolumn{2}{|c|}{0.0151} & \multicolumn{2}{|c|}{0.0019} & \multicolumn{2}{|c|}{0.0056} & \multicolumn{2}{|c|}{0.0038} \\
\hline
\end{tabular}

As shown in Table 6.5, all the p-values illustrate significant scenarios (less than $0.5 \%$, reject $H_{0}$ ). We obtain two conclusions from the above results. (1) Each $p$-value reveals that there is a significant differences between the results obtained by RO model and SPR model. (2) Given the Avg. ranking values, we find the advantage of the indicators. So the solutions obtained by the RO model have more strength in robustness than the solutions obtained by the SPR model.

To sum up, the HHCRSP formulated by RO model shows advantage in robustness than that modeled by SPR.

\section{7. conclusion}

Even though a large amount of work has been done for the HHCRSPs, the majority of them belongs to the deterministic models, which can only make the final decision on the schedule satisfy 
the deterministic scenarios. According to the feedback from HHC companies, caregivers have to deal with some uncertainties when carrying out a given schedule to visit their patients. This work formulates a robust optimization model for an HHC Routing and Scheduling Problem with taking into account uncertain travel and service times. Specifically, the non-deterministic variables are defined based on the theory of budget uncertainty, and then the arrival time of each caregiver is rewritten as a complicated recursive function. After that, Gurobi Solver, Simulated Annealing, Tabu Search, and Variable Neighborhood Search are adapted to solve the model respectively. Finally, a series of experiments have been performed to validate the proposed models and algorithms. Experimental results from Monte Carlo simulation highlight the strength of considering uncertainties when modeling the problem. Additional, the influences of other characters in instances, like the width of time-window, distributed location have also been empirically analyzed.

Finally, the comparison between the solutions obtained by the robust optimization model and stochastic programming with recourse is performed.

The results show that the proposed tabu search is able to produce effective solutions for the generated instances. We conducted a comprehensive analysis of the results, and several insight observations are summarized as follows.

(1) The solution of the scheduling and routing, which obtained by ignoring uncertainty of travel time gives very weak scheduling strategies that often lead to delayed services.

(2) Incorporating more vehicles into a routing program and reorganizing the sequence of customer visits in each vehicle route can sometimes increase the robustness of a given routing strategy.

(3) Extremely robust routing strategies can be generated for parameters characterized by wide time windows for customers and high capacity vehicles at low additional cost.

(4) The instances with patients characterized by narrow time-windows show poor robustness in the deterministic model, and achieving a high level of robustness is much more expensive, it takes more vehicles and much longer distances.

(5) In the model of robust optimizations, the solutions with considering both travel and service time uncertainty show more robustness than those solutions obtained only one uncertainty. However, the solutions considering just one uncertainty, still show advantage than without taking into account any uncertainties.

(6) Even if we consider the uncertainties, the model formulated from robust optimization shows strength in robustness than that modeled by the stochastic model with recourse. 
However, like most of the studies involved robust optimization technique, for example, Hu et al. (2018); Wu et al. (2017), we have also assumed that the uncertainty set is characterized by the theory of budget uncertainty. The parameters related to the uncertainty set were set the same as the published work (Hu et al., 2018; Wu et al., 2017). Our model could be more close to the practice if the parameters are designed from big data (Zhang et al., 2017; Choi et al., 2018) of the HHC industry. So, the integration of robust optimization techniques with big data is one of our future work.

There are also many other interesting extensions to this work worthy of further consideration. On the one hand, it is interesting to extend the problem with considering other practical constraints like lunch-break time of caregivers, and flexible time-window of patients. On the other side, from the perspective of improving solving methods, we can consider the incorporation of machine learning techniques with meta-heuristic to obtain more efficiency solutions (Schindl \& Zufferey, 2015; Chen et al., 2016b). Furthermore, sharing economic (Choi \& He, 2019) also provides a promising way to improve the efficiency of the operational management of HHC service.

\section{Appendix A. Stochastic Programming model with Recourse}

SPR is a common used technique for modeling the optimization problems when considering uncertainties. Anyone who is interested in the modeling of SPR can refer to the published work (Li et al., 2010; Shi et al., 2018; Zhang et al., 2019). The main idea of SPR can be viewed as a two-stage programming problem. Generally, in the first stage, planned routes and scheduling are designed according to the current information; while in the second stage, travel time and service time are realized, the expected penalty caused by delayed service is considered. This appendix mainly introduces the detailed formulations of the SPR model for our HHCRSP.

\section{Appendix A.0.1. Mathematical model}

Before presenting the SPR model, we would like to describe some new notations. Meanwhile, some other notations, which have already been introduced in the RO model part, will be omitted here. 
Table A.10: new notations in the SPR model

\begin{tabular}{ll}
\hline notation & meaning \\
\hline$\mu_{i j}$ & average driving time between node $i$ and $j$ \\
$\sigma_{i j}$ & standard deviation value of the driving time between node $i$ and $j$. \\
$\check{t_{i j}} \sim N\left(\mu_{i j}, \sigma_{i j}^{2}\right)$ & stochastic variable of the travel time between patients $i$ and $j$ for a single trip. \\
$\mu_{i}$ & the average service time for patient $i$ \\
$\sigma_{i}$ & standard deviation of the service time for patient $i$ \\
$\check{t_{i}} \sim N\left(\mu_{i}, \sigma_{i}^{2}\right)$ & stochastic variable of the service time for patient $i$. \\
$\check{\theta_{k}}$ & extra working time for the caregiver of route $k$. \\
$\check{\alpha}$ & unit caregiver remuneration for the extra working time. \\
$\check{\delta_{k}}:$ & the total delayed time for all the patients in route $k$. \\
$\check{\beta}$ & unit penalty cost for driving time after the due time $b_{i}$. \\
$U(\check{E})=\left[u_{1}(\check{E}), u_{2}(\check{E}), \ldots, u_{n+1}(\check{E})\right]^{T}$ is a $(n+1) \times 1$ & coefficient of recourse. \\
$W(\check{E})=\left[w_{1}(\check{E}), w_{2}(\check{E}), \ldots, w_{n+1}(\check{E})\right]^{T}$ is a $(n+1) \times 1$ & the value of a constraint violation. \\
$E(\cdot)$ & the recourse value caused by delayed service and extra working time. \\
$\check{S_{i k}}$ & the beginning service time of patient $i$, who receives service from caregiver $k$. \\
\hline
\end{tabular}

The SPR model can be formulated as:

$$
\min \sigma \cdot \sum_{k \in V} c f_{k} \sum_{j \in C} x_{0 j k}+\sum_{k \in V} \sum_{i \in N} \sum_{j \in N} c_{i j} x_{i j k}+E\left[\min U(\check{E})^{T} W(\check{E})\right]
$$

S.T.

$$
\begin{gathered}
\sum_{k \in V} \sum_{j \in N} x_{i j k}=1, \forall i \in C, \\
\sum_{i \in N} x_{i h k}-\sum_{j \in N} x_{h j k}=0, \forall h \in C ; k \in V, \\
\sum_{j \in C} x_{0 j k} \leq 1, \forall k \in V, \\
\sum_{j \in C} x_{j(n+1) k} \leq 1, \forall k \in V, \\
\sum_{i \in N} \sum_{j \in N} x_{i j k} \leq Q, \forall k \in V
\end{gathered}
$$




$$
\begin{aligned}
& d_{i} \sum_{j \in N} x_{i j k} \leq D_{k}, \forall k \in V, i \in C, \\
& \check{s_{i k}}+\check{t_{i}}+\check{t_{i j}}-M\left(1-x_{i j k}\right) \leq \check{s_{j k}}, \forall i, j \in N ; k \in V, \\
& a_{i} \leq \check{s_{i k}}, \forall i \in N ; k \in V, \\
& \check{s_{i k}}-w_{i}(\check{E}) \leq b_{i}, \forall i \in N, k \in V \\
& x_{i j k} \in\{0,1\}, y_{i j} \geq 0, z_{i j} \geq 0, w_{i}(\check{E}) \geq 0, \forall i, j \in N, k \in V,
\end{aligned}
$$

The objective function (A.1) is to minimize the total cost, which includes the salary of caregivers, transportation cost, and the expected value of recourse value caused by delayed service (the detailed description is shown in Appendix A.1). Constraints (A.2)-(A.7) has already been explained in the RO model, therefore, we omit the explanation. Constraints (A.8)-(A.10) are the time window constraints. Finally, constraints (A.11) define the nature of the decision variables.

Remark As a special scenario, if $U(\check{E})=\left[u_{1}(\check{E}), u_{2}(\check{E}), \ldots, u_{n+1}(\check{E})\right]$ is a $(n+1) \times 1$ becomes a deterministic value, the model is called a Stochastic Programming with Fixed Recourse. In this paper, we assume that, if $i=n+1, u_{i}(\check{E})=\check{\beta}$, otherwise, $u_{i}(\check{E})=\check{\alpha}$. This assumptions has also been adopted by Li et al. (2010); Shi et al. (2018)

\section{Appendix A.1. Recourse of the model}

The recourse part in stochastic programming is generally formulated by altering the hard constraints to the corresponding soft ones. According to the characteristic of our problem (Li et al., 2010; Shi et al., 2018), the constraints related to travel and service times should be relaxed to soft ones.

Furthermore, constraints (A.10) reveal that a recourse will be produced once a time window constraint is violated. The recourse formulation includes two aspects as follows.

(1) When $i=1, \ldots, n, \min w_{i}(\check{E})$ indicates that the delayed service has been provided for patient $i$. 
(2) When $i=n+1, \min w_{i}(\check{E})$ reveals that the caregiver finishes his/her task later than the due closing time $b_{n+1}$. In this scenario, caregivers have to deal with a extra working time for finishing task.

For a given route $k \in V$, let $r_{k}$ represent the set of nodes in route $k$, and $n_{k}=\left|r_{k}\right|$ be the number of nodes in route $k . r_{k}$ can be written as $r_{k}=\left\{v_{0}=\operatorname{depot}, v_{1}, \ldots, v_{j}, \ldots v_{n_{k}}, v_{n_{k}+1}=\right.$ lab $\}$, in which $v_{j}$ represents the $j$ th patient in this route. According to the time window constraint, it must hold $\check{S}_{v_{j+1}}=\max \left(\check{S}_{v_{j}}+\check{t}_{v_{j} v_{j+1}}+\check{t}_{v_{j}}, a_{v_{j+1}}\right)$. The total delayed time for the route $k$ can be computed as:

$$
\check{\delta_{k}}=\sum_{j \in r_{k}} \max \left(\check{S_{v_{j}}}-b_{r_{j}}, 0\right)
$$

Now, the extra working time for each caregiver can be computed by the following formulation.

$$
\check{\theta_{k}}=\check{S}_{v_{n_{k}+1}}-b_{n+1}
$$

Finally, the recourse part of the stochastic programming is calculated as follows.

$$
\min U(\check{E})^{T} W(\check{E})=\sum_{k \in V}\left[\check{\alpha} \cdot \check{\theta_{k}}+\check{\beta} \cdot \check{\delta_{k}}\right]
$$

The recourse formulation (A.14) is estimated by the Monte Carlo Simulation, which is shown in Figure 5. To solve the problem, we adopt the TS-based heuristic algorithm. The detailed procedures can refer to Li et al. (2010).

\section{Appendix B. Experimental results}

The tables of results are shown in landscape style of the next pages. 


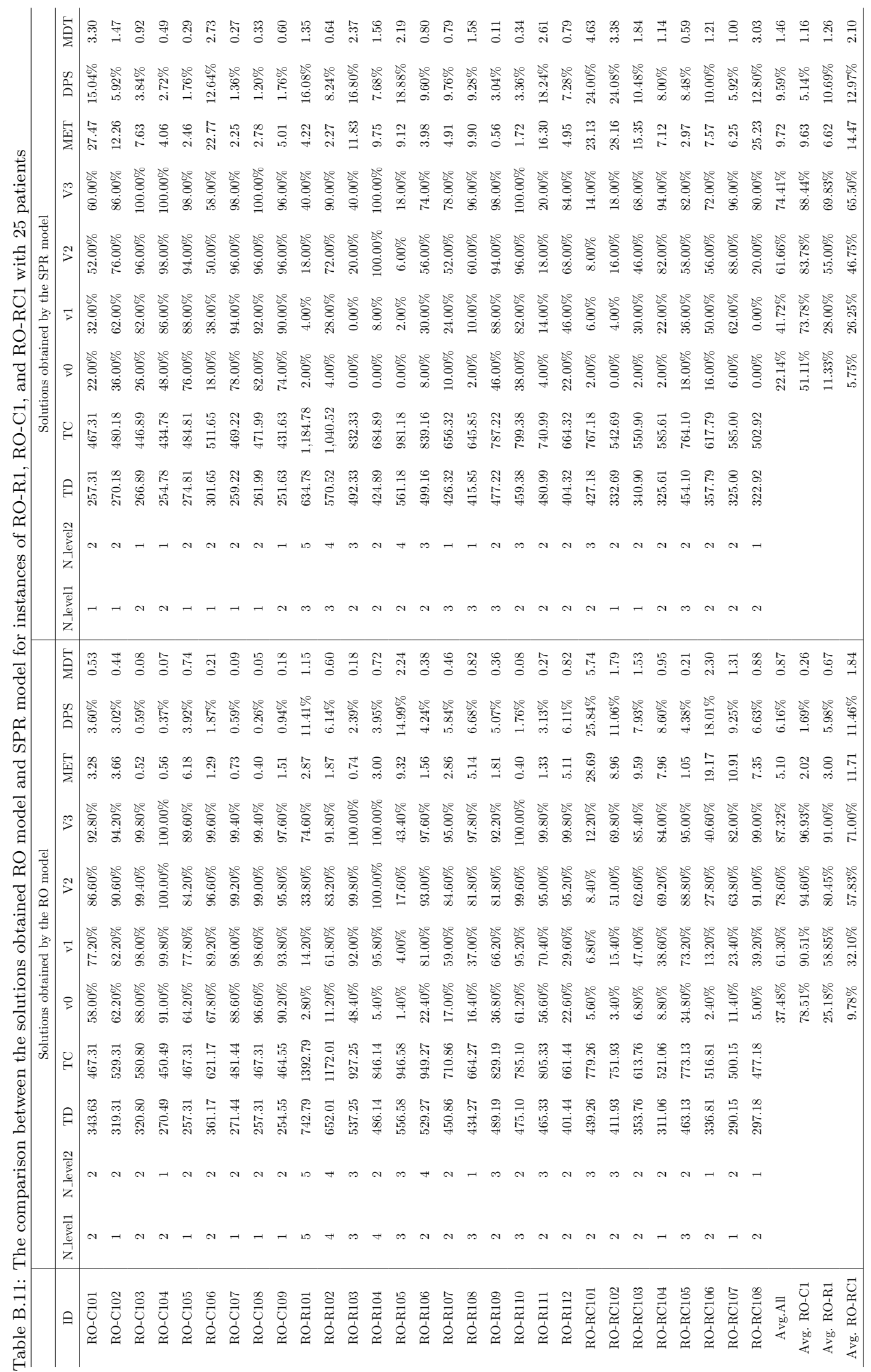




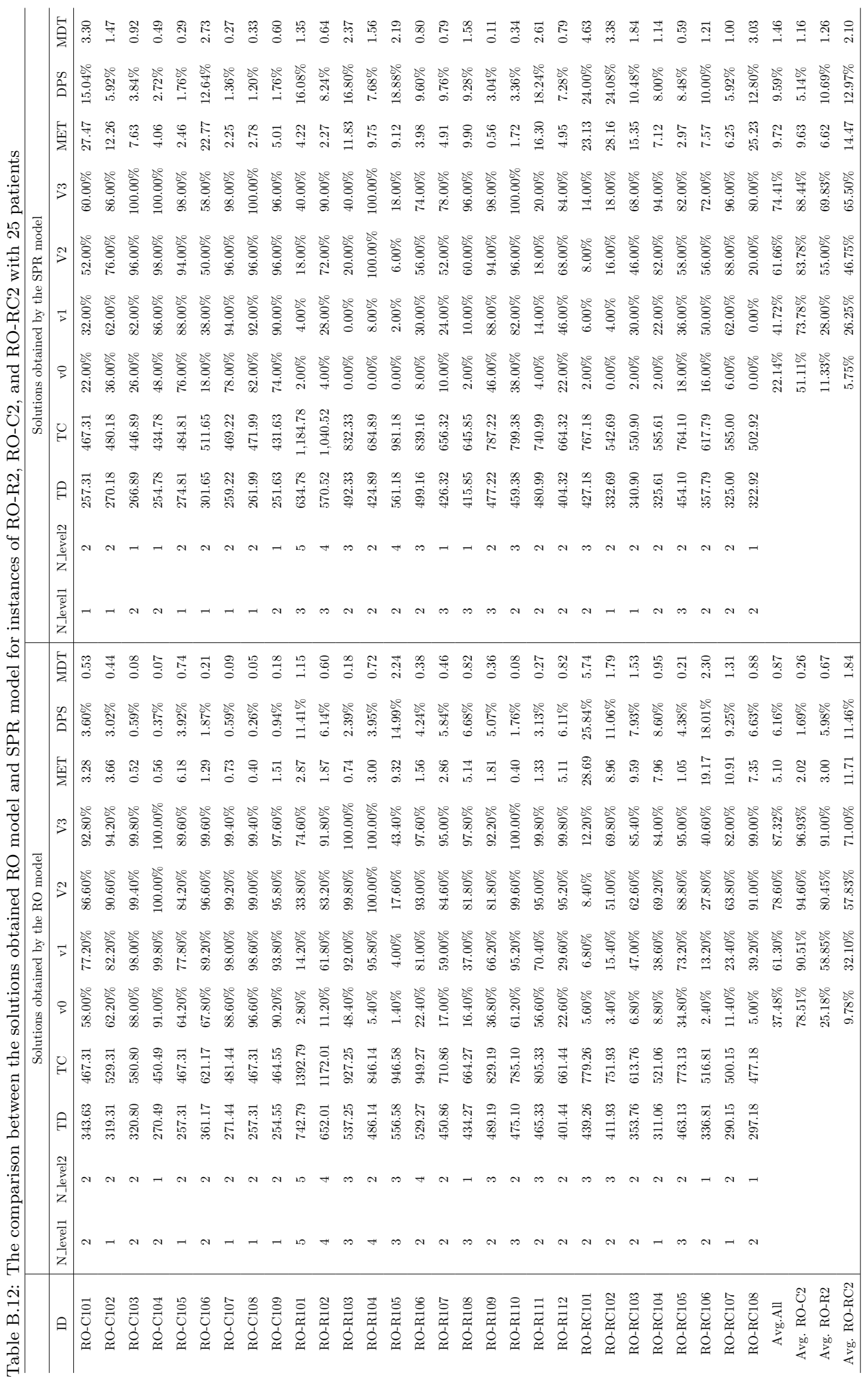




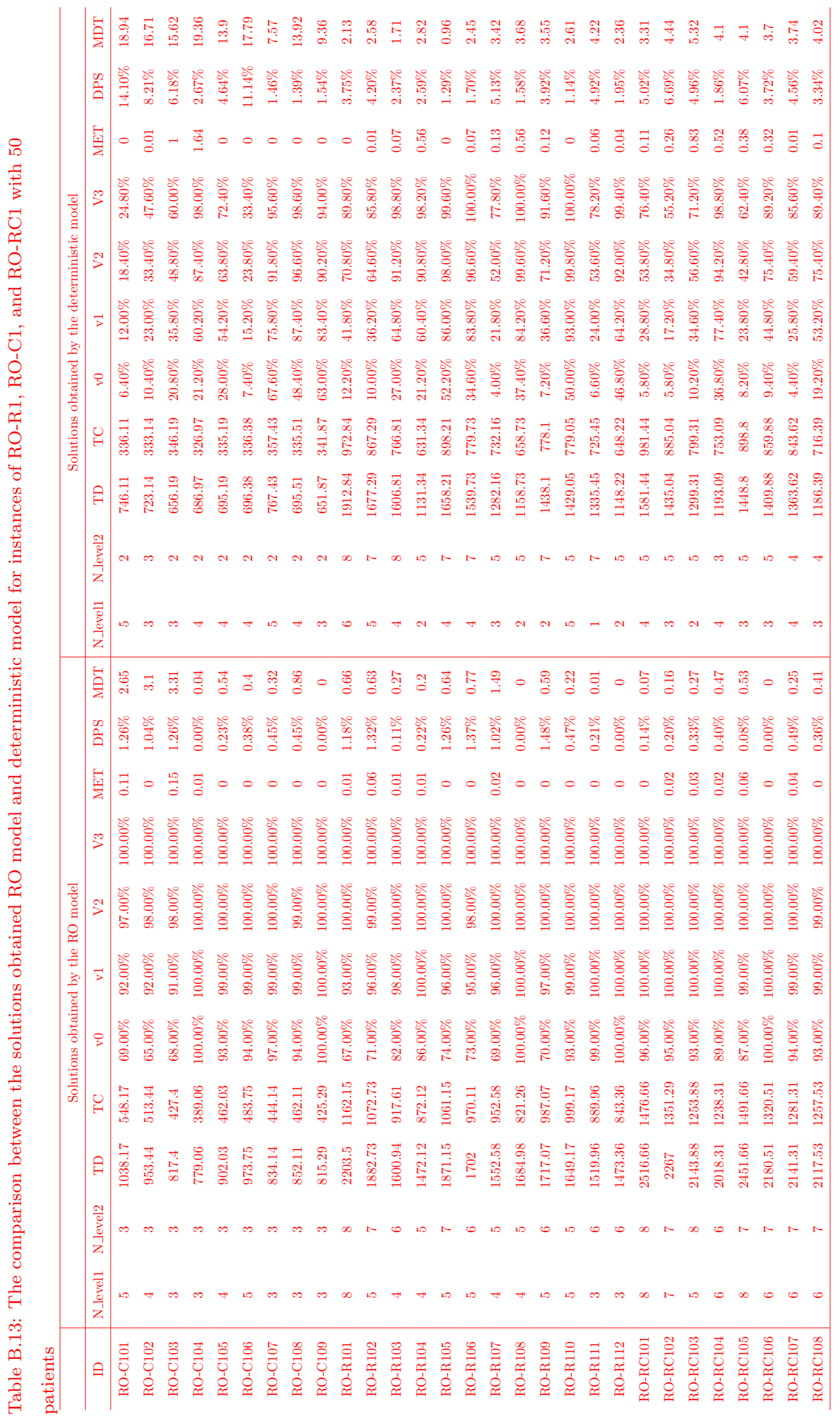




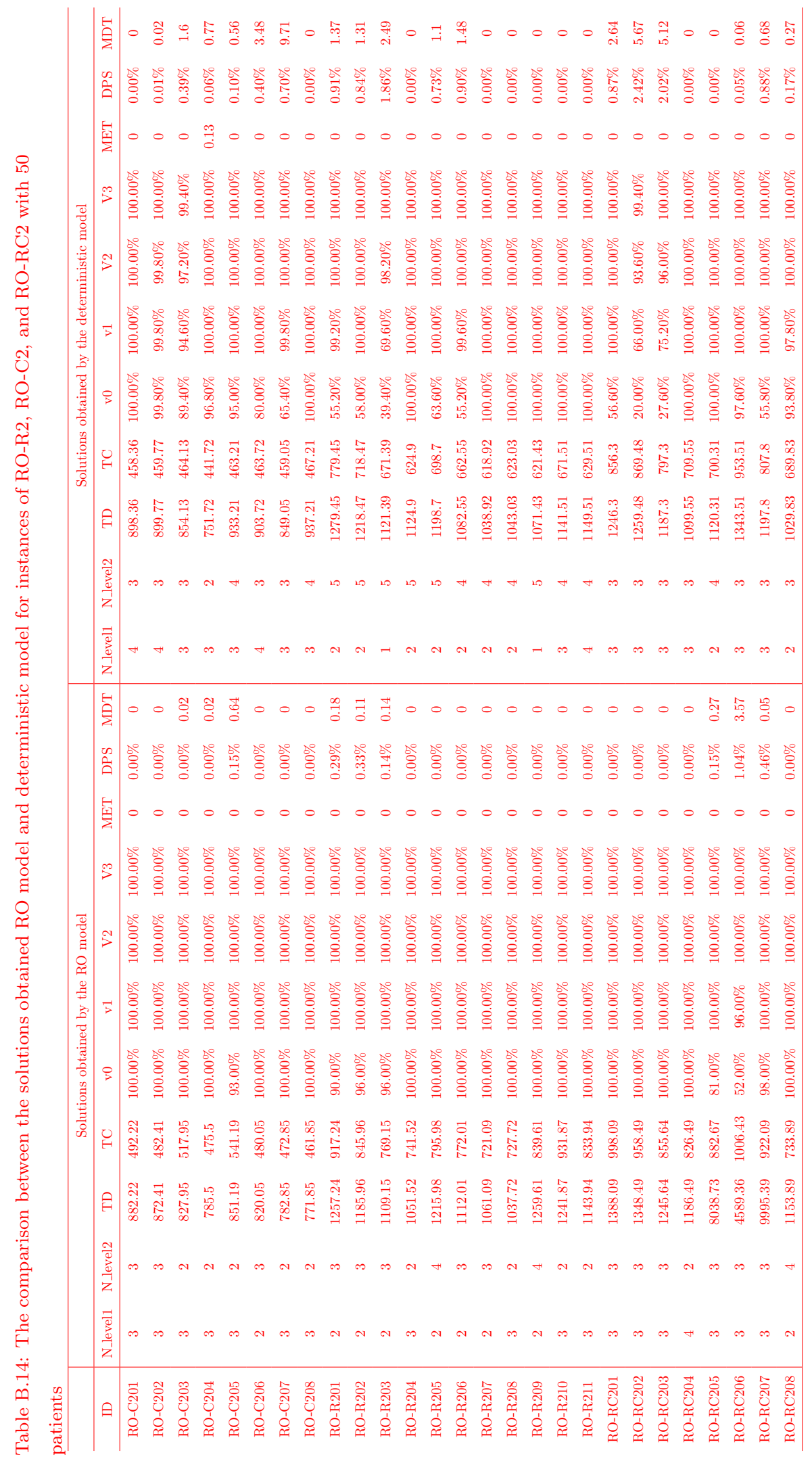




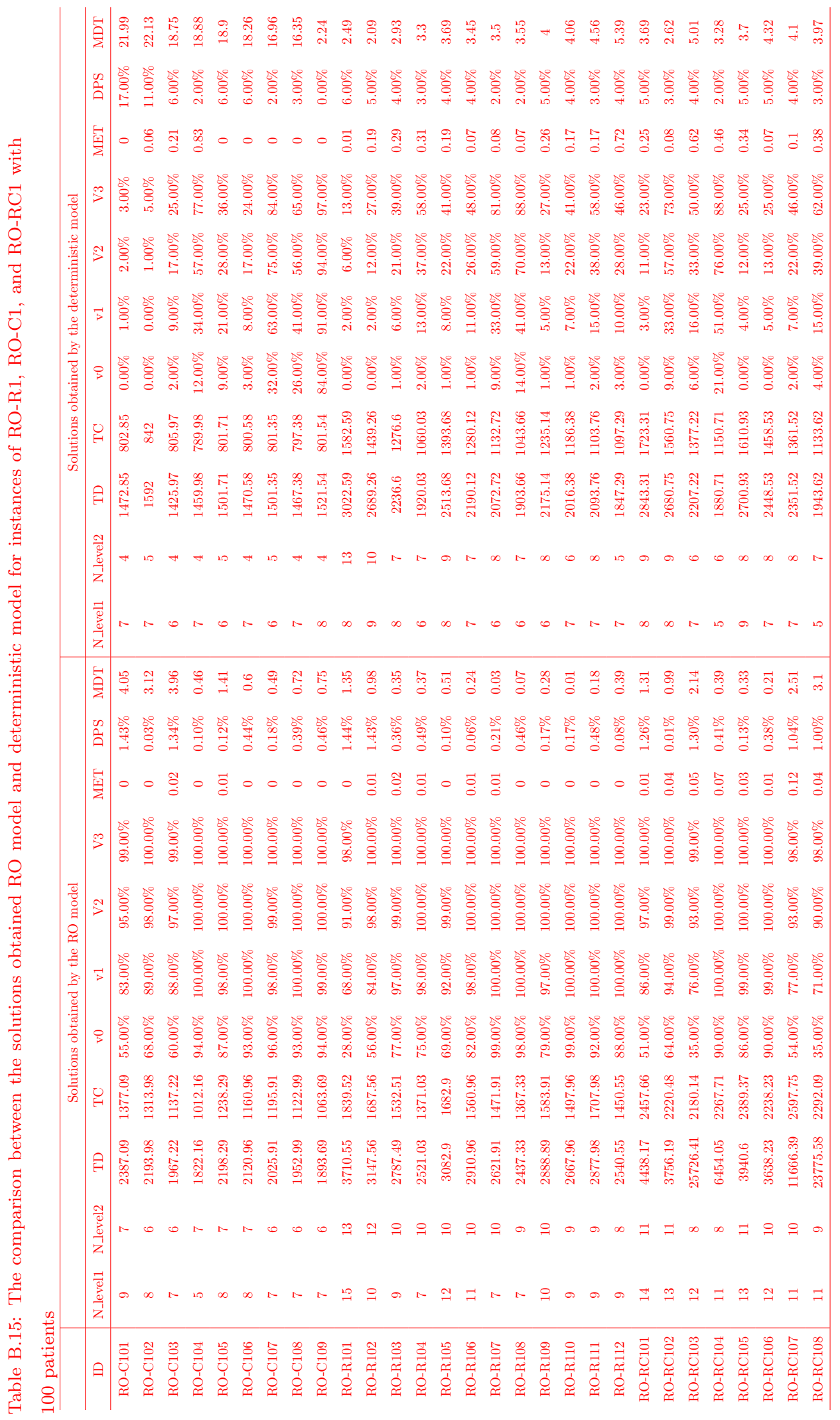




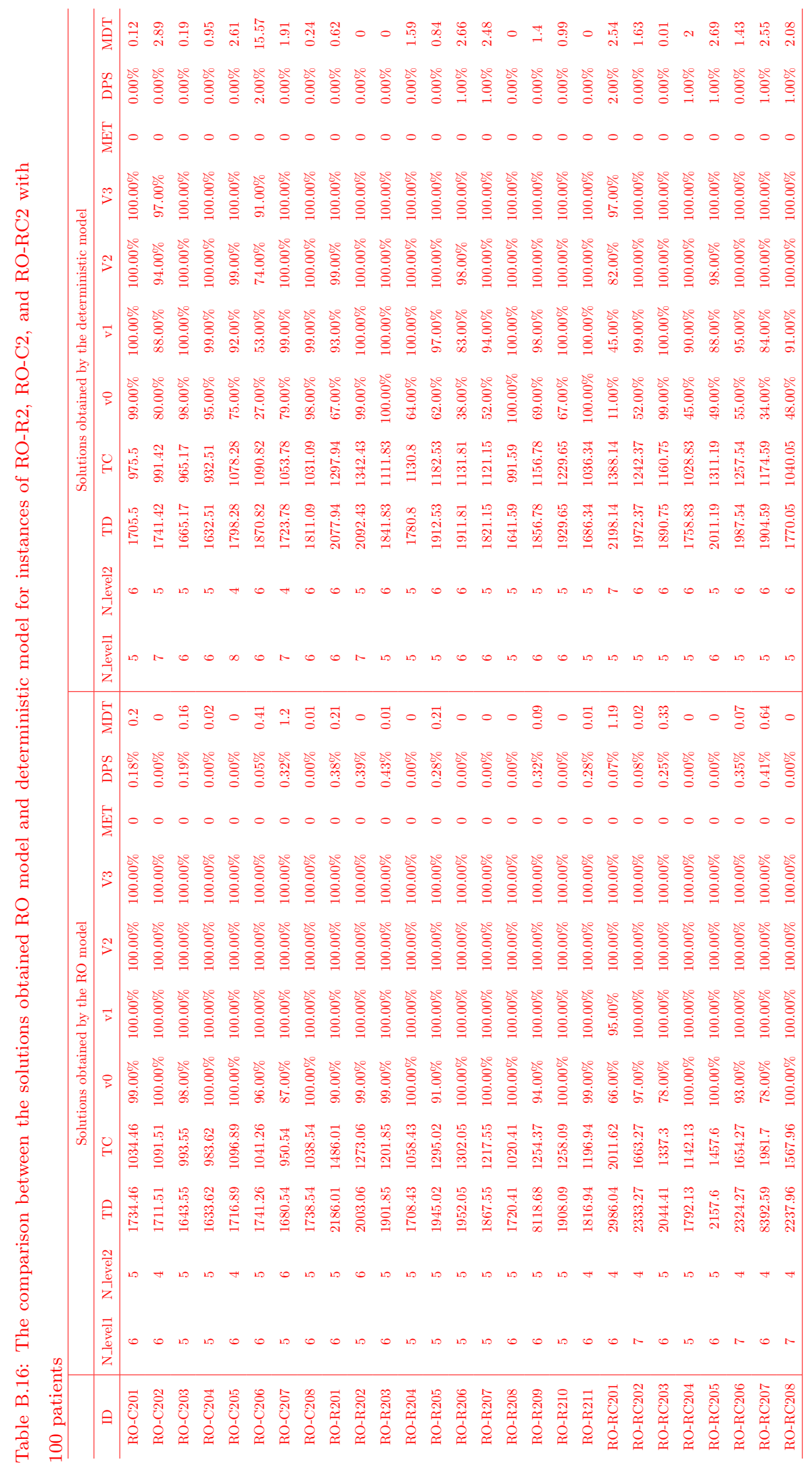




\section{References}

Adulyasak, Y., \& Jaillet, P. (2015). Models and algorithms for stochastic and robust vehicle routing with deadlines. Transportation Science, 50, 608-626.

Agra, A., Christiansen, M., Figueiredo, R., Hvattum, L. M., Poss, M., \& Requejo, C. (2013). The robust vehicle routing problem with time windows. Computers $\& 3$ operations research, 40 , 856-866.

Akjiratikarl, C., Yenradee, P., \& Drake, P. R. (2007). Pso-based algorithm for home care worker scheduling in the uk. Computers \& Industrial Engineering, 53, 559-583.

Alodhayani, A. A. (2017). Comparison between home health care and hospital services in elder population: cost-effectiveness. Biomedical Research, 28, 2087-2090.

Begur, S. V., Miller, D. M., \& Weaver, J. R. (1997). An integrated spatial dss for scheduling and routing home-health-care nurses. Interfaces, 27, 35-48.

Ben-Tal, A., \& Nemirovski, A. (1999). Robust solutions of uncertain linear programs. Operations research letters, 25, 1-13.

Bortfeldt, A., Hahn, T., Männel, D., \& Mönch, L. (2015). Hybrid algorithms for the vehicle routing problem with clustered backhauls and 3d loading constraints. European Journal of Operational Research, 243, 82-96.

Braaten, S., Gjønnes, O., Hvattum, L. M., \& Tirado, G. (2017). Heuristics for the robust vehicle routing problem with time windows. Expert Systems with Applications, 77, 136-147.

Chen, L., Gendreau, M., Hà, M. H., \& Langevin, A. (2016a). A robust optimization approach for the road network daily maintenance routing problem with uncertain service time. Transportation research part E: logistics and transportation review, 85, 40-51.

Chen, M.-C., Hsiao, Y.-H., Reddy, R. H., \& Tiwari, M. K. (2016b). The self-learning particle swarm optimization approach for routing pickup and delivery of multiple products with material handling in multiple cross-docks. Transportation Research Part E: Logistics and Transportation Review, 91, 208-226. 
Choi, T.-M., \& He, Y. (2019). Peer-to-peer collaborative consumption for fashion products in the sharing economy: Platform operations. Transportation Research Part E: Logistics and Transportation Review, 126, 49-65.

Choi, T.-M., Wallace, S. W., \& Wang, Y. (2018). Big data analytics in operations management. Production and Operations Management, 27, 1868-1883.

Cordeau, J.-F., Laporte, G., \& Mercier, A. (2001). A unified tabu search heuristic for vehicle routing problems with time windows. Journal of the Operational research society, 52, 928-936.

Decerle, J., Grunder, O., Hassani, A. H. E., \& Barakat, O. (2018). A memetic algorithm for multiobjective optimization of the home health care problem. Swarm and Evolutionary Computation, . doi:https ://doi.org/10.1016/j.swevo.2018.08.014.

Derrac, J., García, S., Molina, D., \& Herrera, F. (2011). A practical tutorial on the use of nonparametric statistical tests as a methodology for comparing evolutionary and swarm intelligence algorithms. Swarm and Evolutionary Computation, 1, 3-18.

Di Mascolo, M., Espinouse, M.-L., \& El Hajri, Z. (2017). Planning in home health care structures: A literature review. IFAC-PapersOnLine, 50, 4654-4659.

Escobar, J. W., Linfati, R., Toth, P., \& Baldoquin, M. G. (2014). A hybrid granular tabu search algorithm for the multi-depot vehicle routing problem. Journal of Heuristics, 20, 483-509.

Eshtehadi, R., Fathian, M., Pishvaee, M. S., \& Demir, E. (2018). A hybrid metaheuristic algorithm for the robust pollution-routing problem. Journal of Industrial and Systems Engineering, 11, $0-0$.

Fathollahi-Fard, A. M., Hajiaghaei-Keshteli, M., \& Tavakkoli-Moghaddam, R. (2018). A biobjective green home health care routing problem. Journal of Cleaner Production, 200, 423 - 443. doi:https://doi.org/10.1016/j.jclepro.2018.07.258.

Fikar, C., \& Hirsch, P. (2017). Home health care routing and scheduling: A review. Computers $\mathbb{E}$ Operations Research, 77, 86-95.

Friedman, M. (1940). A comparison of alternative tests of significance for the problem of $m$ rankings. The Annals of Mathematical Statistics, 11, 86-92. 
Garcia, B.-L., Potvin, J.-Y., \& Rousseau, J.-M. (1994). A parallel implementation of the tabu search heuristic for vehicle routing problems with time window constraints. Computers 83 Operations Research, 21, 1025-1033.

Glover, F. (1986). Future paths for integer programming and links to artificial intelligence. Computers $\&$ operations research, 13, 533-549.

Hu, C., Lu, J., Liu, X., \& Zhang, G. (2018). Robust vehicle routing problem with hard time windows under demand and travel time uncertainty. Computers $\&$ Operations Research, 94, 139-153.

Kuo, R., Wibowo, B., \& Zulvia, F. (2016). Application of a fuzzy ant colony system to solve the dynamic vehicle routing problem with uncertain service time. Applied Mathematical Modelling, 40, 9990-10001.

Lai, D. S., Demirag, O. C., \& Leung, J. M. (2016). A tabu search heuristic for the heterogeneous vehicle routing problem on a multigraph. Transportation Research Part E: Logistics and Transportation Review, 86, 32-52.

Lanzarone, E., \& Matta, A. (2014). Robust nurse-to-patient assignment in home care services to minimize overtimes under continuity of care. Operations Research for Health Care, 3, 48-58.

Li, H., Jian, X., Chang, X., \& Lu, Y. (2018). The generalized rollon-rolloff vehicle routing problem and savings-based algorithm. Transportation Research Part B: Methodological, 113, 1-23.

Li, X., Tian, P., \& Leung, S. C. (2010). Vehicle routing problems with time windows and stochastic travel and service times: Models and algorithm. International Journal of Production Economics, $125,137-145$.

Liu, R., Xie, X., Augusto, V., \& Rodriguez, C. (2013). Heuristic algorithms for a vehicle routing problem with simultaneous delivery and pickup and time windows in home health care. European Journal of Operational Research, 230, 475-486.

Liu, R., Xie, X., \& Garaix, T. (2014). Hybridization of tabu search with feasible and infeasible local searches for periodic home health care logistics. Omega, 47, 17-32.

Liu, R., Yuan, B., \& Jiang, Z. (2018). A branch-and-price algorithm for the home-caregiver schedul${ }_{885} \quad$ ing and routing problem with stochastic travel and service times. Flexible Services and Manufacturing Journal, (pp. 1-23). 
Luo, Z., Qin, H., Zhang, D., \& Lim, A. (2016). Adaptive large neighborhood search heuristics for the vehicle routing problem with stochastic demands and weight-related cost. Transportation Research Part E: Logistics and Transportation Review, 85, 69-89.

Mankowska, D. S., Meisel, F., \& Bierwirth, C. (2014). The home health care routing and scheduling problem with interdependent services. Health care management science, 17, 15-30.

Nickel, S., Schröder, M., \& Steeg, J. (2012). Mid-term and short-term planning support for home health care services. European Journal of Operational Research, 219, 574-587.

Osaba, E., Yang, X.-S., Diaz, F., Lopez-Garcia, P., \& Carballedo, R. (2016). An improved discrete bat algorithm for symmetric and asymmetric traveling salesman problems. Engineering Applications of Artificial Intelligence, 48, 59-71.

Osaba, E., Yang, X.-S., Fister Jr, I., Del Ser, J., Lopez-Garcia, P., \& Vazquez-Pardavila, A. J. (2019). A discrete and improved bat algorithm for solving a medical goods distribution problem with pharmacological waste collection. Swarm and evolutionary computation, 44, 273-286.

Rodriguez, C., Garaix, T., Xie, X., \& Augusto, V. (2015). Staff dimensioning in homecare services with uncertain demands. International Journal of Production Research, 53, 7396-7410.

Sarasola, B., Doerner, K. F., Schmid, V., \& Alba, E. (2016). Variable neighborhood search for the stochastic and dynamic vehicle routing problem. Annals of Operations Research, 236, 425-461.

Schindl, D., \& Zufferey, N. (2015). A learning tabu search for a truck allocation problem with linear and nonlinear cost components. Naval Research Logistics (NRL), 62, 32-45.

Shi, Y., Boudouh, T., \& Grunder, O. (2017). A hybrid genetic algorithm for a home health care routing problem with time window and fuzzy demand. Expert Systems with Applications, 72, $160-176$.

Shi, Y., Boudouh, T., Grunder, O., \& Wang, D. (2018). Modeling and solving simultaneous delivery and pick-up problem with stochastic travel and service times in home health care. Expert Systems with Applications, 102, 218-233.

Silvestrin, P. V., \& Ritt, M. (2017). An iterated tabu search for the multi-compartment vehicle routing problem. Computers \& Operations Research, 81, 192-202. 
Solano-Charris, E., Prins, C., \& Santos, A. C. (2015). Local search based metaheuristics for the robust vehicle routing problem with discrete scenarios. Applied Soft Computing, 32, 518-531.

Solomon, M. M. (1987). Algorithms for the vehicle routing and scheduling problems with time window constraints. Operations research, 35, 254-265.

Sun, W., Yu, Y., \& Wang, J. (2019). Heterogeneous vehicle pickup and delivery problems: Formulation and exact solution. Transportation Research Part E: Logistics and Transportation Review, 125, 181-202. URL: http://www.sciencedirect.com/science/article/pii/ S1366554519301267. doi:https://doi.org/10.1016/j.tre.2019.03.012.

Taillard, É., Badeau, P., Gendreau, M., Guertin, F., \& Potvin, J.-Y. (1997). A tabu search heuristic for the vehicle routing problem with soft time windows. Transportation science, 31, 170-186.

Tyan, M. (2010). Understanding Taiwanese home healthcare nurse managers' empowerment and international learning experiences: Community-based participatory research approach using a US home healthcare learning tour. University of Washington.

Wang, C., Mu, D., Zhao, F., \& Sutherland, J. W. (2015). A parallel simulated annealing method for the vehicle routing problem with simultaneous pickup-delivery and time windows. Computers E Industrial Engineering, 83, 111-122.

Wang, J., Yu, Y., \& Tang, J. (2018). Compensation and profit distribution for cooperative green pickup and delivery problem. Transportation Research Part B: Methodological, 113, 54-69. URL: http://www.sciencedirect.com/science/article/pii/S0191261517302825. doi:https://doi.org/10.1016/j.trb.2018.05.003.

Wu, L., Hifi, M., \& Bederina, H. (2017). A new robust criterion for the vehicle routing problem with uncertain travel time. Computers $\&$ Industrial Engineering, 112, 607-615.

Yu, Y., Wang, S., Wang, J., \& Huang, M. (2019). A branch-and-price algorithm for the heterogeneous fleet green vehicle routing problem with time windows. Transportation Research Part B: Methodological, 122, 511-527. URL: http://www.sciencedirect.com/science/article/pii/ S0191261518308944. doi:https://doi.org/10.1016/j.trb.2019.03.009. 
Yuan, B., Liu, R., \& Jiang, Z. (2015). A branch-and-price algorithm for the home health care scheduling and routing problem with stochastic service times and skill requirements. International Journal of Production Research, 53, 7450-7464.

Zafra, A., Gibaja, E. L., \& Ventura, S. (2011). Multiple instance learning with multiple objective genetic programming for web mining. Applied Soft Computing, 11, 93-102.

Zhang, C., Liu, C., Zhang, X., \& Almpanidis, G. (2017). An up-to-date comparison of state-of-theart classification algorithms. Expert Systems with Applications, 82, 128-150.

Zhang, J., Dridi, M., \& El Moudni, A. (2019). A two-level optimization model for elective surgery scheduling with downstream capacity constraints. European Journal of Operational Research, . doi:https://doi.org/10.1016/j.ejor.2019.01.036. 